\title{
Basal Ganglia Outputs Map Instantaneous Position Coordinates during Behavior
}

\author{
Joseph W. Barter, ${ }^{1,3}$ CSuellen Li, ${ }^{1}$ Tatyana Sukharnikova, ${ }^{1}$ Mark A. Rossi, ${ }^{1}$ Ryan A. Bartholomew, ${ }^{1}$ and Henry H. Yin ${ }^{1,2,3}$ \\ ${ }^{1}$ Department of Psychology and Neuroscience, ${ }^{2}$ Department of Neurobiology, and ${ }^{3}$ Center for Cognitive Neuroscience, Duke University, Durham, North \\ Carolina 27708
}

The basal ganglia (BG) are implicated in many movement disorders, yet how they contribute to movement remains unclear. Using wireless in vivo recording, we measured $\mathrm{BG}$ output from the substantia nigra pars reticulata $(\mathrm{SNr})$ in mice while monitoring their movements with video tracking. The firing rate of most nigral neurons reflected Cartesian coordinates (either $x$ - or $y$-coordinates) of the animal's head position during movement. The firing rates of $\mathrm{SNr}$ neurons are either positively or negatively correlated with the coordinates. Using an egocentric reference frame, four types of neurons can be classified: each type increases firing during movement in a particular direction (left, right, up, down), and decreases firing during movement in the opposite direction. Given the high correlation between the firing rate and the $x$ and $y$ components of the position vector, the movement trajectory can be reconstructed from neural activity. Our results therefore demonstrate a quantitative and continuous relationship between BG output and behavior. Thus, a steady BG output signal from the $\mathrm{SNr}$ (i.e., constant firing rate) is associated with the lack of overt movement, when a stable posture is maintained by structures downstream of the BG. Any change in $\mathrm{SNr}$ firing rate is associated with a change in position (i.e., movement). We hypothesize that the SNr output quantitatively determines the direction, velocity, and amplitude of voluntary movements. By changing the reference signals to downstream position control systems, the BG can produce transitions in body configurations and initiate actions.

Key words: basal ganglia; GABA; Parkinson’s disease; substantia nigra

\section{Introduction}

The basal ganglia (BG) receive excitatory inputs from the cerebral cortex and thalamus and send inhibitory outputs to the brainstem, tectum, and diencephalon. They have been implicated in learning, habit formation, and reward (Graybiel, 1998; Yin and Knowlton, 2006), yet they also play a major role in motor control (DeLong et al., 1984; Romo and Schultz, 1992). Although disorders of the BG (e.g., Parkinson's disease) are characterized by severe motor deficits, it remains unclear how the BG output generates movements.

According to the most popular model of BG function, a decrease in the inhibitory BG output enables movements, whereas an increase in the output prevents movements (Albin et al., 1989; DeLong, 1990; Hikosaka et al., 2000). Although this model is supported by a number of studies (Hikosaka et al., 2000), other observations seem to be incompatible with the disinhibition hypothesis. For example, neurons in the substantia nigra pars reticulata ( $\mathrm{SNr}$, a major BG output nucleus) are known to both increase and decrease their firing rates in relation to movement

\footnotetext{
Received Aug. 5, 2014; revised Nov. 26, 2014; accepted Dec. 17, 2014.

Author contributions: J.W.B. and H.H.Y. designed research; J.W.B., S.L., T.S., M.A.R., and R.A.B. performed research; J.W.B. and H.H.Y. analyzed data; J.W.B. and H.H.Y. wrote the paper.

This work was supported by National Institutes of Health Grant 021074 to H.H.Y. and National Science Foundation graduate fellowships to J.W.B. and M.A.R.

The authors declare no competing financial interests.

Correspondence should be addressed to Dr. Henry H. Yin, Box 91050, Duke University, Durham, NC 27708. E-mail: hy43@duke.edu.

DOI:10.1523/JNEUROSCI.3245-14.2015

Copyright $\odot 2015$ the authors $\quad 0270-6474 / 15 / 352703-14 \$ 15.00 / 0$
}

(Gulley et al., 1999; Basso and Wurtz, 2002; Fan et al., 2012; Freeze et al., 2013). To explain the opponent outputs, it has been argued that the BG output disinhibits some actions while inhibiting competing actions (Mink, 1996; Cui et al., 2013). But actions are not clearly defined in this "focused selection" model, which assumes that the role of the BG in action selection is all or none, and that the output from the BG does not contribute to movement kinematics.

Recently, we found that putative GABAergic output neurons from the $\mathrm{SNr}$ were significantly modulated by postural disturbance, while tilting the mouse along the roll axis (Barter et al., 2014). Some neurons increased firing when the mouse was tilted to its left and decreased firing when tilted to its right, whereas other neurons displayed the opposite pattern. During postural disturbances, the motor system must generate continuously varying outputs to oppose the effects of tilt on the body configuration. These results suggest that the function of the BG output is not to either inhibit or disinhibit behavior per se, but to send opponent outputs for postural adjustment movements in any direction. Nevertheless, it remains unclear what these opponent signals represent because their quantitative relationship with the actual movement kinematics is unknown.

A major limitation of previous studies is the lack of continuous behavioral measurements and simultaneous recording of movement kinematics and neural activity in unrestrained animals. In this study, we used wireless recording to measure $\mathrm{SNr}$ activity while monitoring movements with video tracking. We designed a simple behavioral task, in which unrestrained mice 
were trained to move repeatedly on a covered and elevated platform, which only prevented locomotion by virtue of its small size. We quantified the head position by tracking a small LED placed on the headstage, without the constraint of cables or head fixation. This design also minimizes movement in the $z$-axis, making it possible to track the movement with a single camera directly facing the mouse.

\section{Materials and Methods}

Subjects and surgery. All procedures were approved by the Duke University Institutional Animal Care and Use Committee. Ten male C57BL6/J mice (25-31 g) were used in the experiments. Detailed procedures for surgeries have been described previously (Fan et al., 2011, 2012; Rossi et al., 2013a). Sixteenchannel electrode arrays (Innovative Neurophysiology) were lowered at the following stereotaxic coordinates in relation to bregma: $2.9-3.0 \mathrm{~mm}$ posterior, $1.2 \mathrm{~mm}$ lateral, and $4.6-4.7 \mathrm{~mm}$ below brain surface ( 5 mice were implanted in the left $\mathrm{SNr} ; 5$ in the right $\mathrm{SNr}$ ). The arrays consisted of micro-polished tungsten wires, $35 \mu \mathrm{m}$ in diameter and $7 \mathrm{~mm}$ in length, arranged in a $4 \times 4$ configuration. All arrays were attached to an Omnetics connector and fixed to the skull with dental acrylic. Row spacing was $200 \mu \mathrm{m}$, and electrode spacing was $150 \mu \mathrm{m}$. Following the completion of the experiments, all mice were perfused and their brains sliced with a Vibratome into $100 \mu \mathrm{m}$ coronal sections, stained with thionin, and examined under a microscope to verify electrode placement.

Behavioral task. To make mice perform reliable movements repeatedly, we gave them limited access to water. After the recording session each day, they had free access to water for $1 \mathrm{~h}$. Each mouse received $\sim 0.5$ $\mathrm{ml}$ to $1.3 \mathrm{ml}$ of $10 \%$ sucrose during the experimental session. When they had free access to water afterward, they consumed $\sim 2 \mathrm{ml}$. The health of the mice was monitored daily by the laboratory staff and veterinarians from the Division of Laboratory Animal Resources at Duke University. On weekends, all mice were given continuous free access to water.

During the session, mice could obtain $10 \%$ sucrose solution from a spout located next to the platform. They perched on a platform positioned within a tube $(5 \mathrm{~cm}$ diameter, $3.8 \mathrm{~cm}$ length, elevated $40 \mathrm{~cm}$ ). The platform $(13 \mathrm{~cm})$ was suspended by a custom built rigid "T"-shaped structure formed of 1-cm-diameter aluminum rod. Mice were first exposed to the elevated platform for at least $1 \mathrm{~h}$ before the start of the experiments, allowing them to acclimate to the experimental setup.

In most experiments. we used a simple reward-guided task, in which sucrose solution was periodically delivered into a spout just below the platform. Each trial began with the presentation of a tone $(21.6 \mathrm{~dB})$ followed by the delivery of $13 \mu \mathrm{l} 10 \%$ sucrose solution dispensed by a Valvelink 8.2 (AutoMate Scientific, click of the solenoid: $34.7 \mathrm{~dB}$ ) and delivered through a rubber tube terminating in a metal spout. On some sessions, the spout was moved laterally to different positions along the front bottom of the platform. The approximate locations of the spout (distance from the edge of the frame in $\mathrm{mm}$ ) were as follows: 40,66 , and 86 ( $x$-axis), and 19 ( $y$-axis).

On some sessions, a $100 \mathrm{~ms}$ tone was used as a cue, and the sucrose was delivered $\sim 2 \mathrm{~s}$ after the termination of the tone (trace conditioning). On other sessions, a 2 s tone was used, and the sucrose was delivered immediately after cue termination. Each session contained 30-250 trials, with an intertrial interval of $20-50 \mathrm{~s}$. Because the variation in the cue duration did not have any effect on the relationship between position coordinates and neural activity, the data were combined in the analysis.

Typically, a mouse would move its entire body after the cue presentation and upon reward delivery. Its body was not restrained, but the size of
B
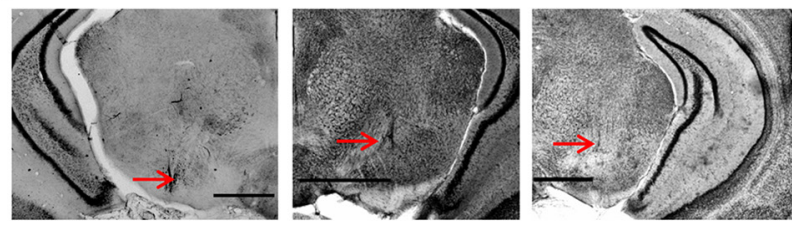

D

$E$
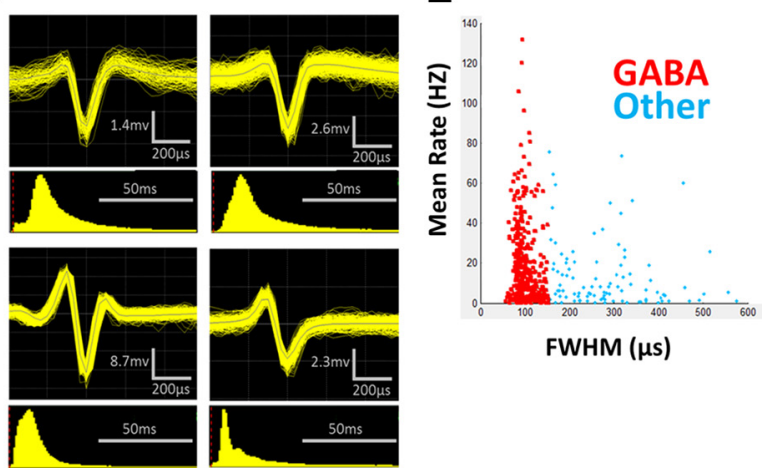

FWHM ( $\mu s)$

Figure 1. In vivo electrophysiological recording and classification of single units. $\boldsymbol{A}$, Illustration of the representative placement (hergic neurons, showing the waveforms and interspike interval distribution. $\boldsymbol{E}$, Plot of firing rate versus spike width. Neurons excluded (other).

the tube prevented it from turning around. The mouse could readily move toward the spout to collect sucrose reward and move back to resume its resting position.

In some experiments ( 4 mice), we used both sucrose and air puff trials. The introduction of aversive air puff trials is designed to test whether the observed correlation between neural activity and movement kinematics is independent of the valence of the outcome. For sessions comparing reward trials and air puff trials, either an $\mathrm{ABAB}$ design was used, in which reward trials and air puff trials were presented in alternate blocks, or an $\mathrm{AB}$ design was used, in which air puff trials were followed by reward trials. The cue duration for these sessions was always $100 \mathrm{~ms}$. Air puffs $(82.7 \mathrm{kPa})$ were $200 \mathrm{~ms}$ in duration and delivered from a computercontrolled 1500 series dispenser (EFD) from $10 \mathrm{~cm}$ below the animal.

Video tracking. Mouse movement was recorded with a steady-frame rate camera (Sony Handicam HRD-XR160) at 30 frames/s. Video and neural datasets were aligned with an LED flash at the start of each session, which generated a video time-marker in synchrony with a transistortransistor logic pulse in the Cerebus data acquisition system (Blackrock). Mouse movement was digitized offline using Optimap (Triangle BioSystems International), which tracked LED position in each frame in pixel coordinates. Because of the orientation of the camera and the fact that the mouse is prevented from significantly changing orientation, these pixel coordinates accurately reflect head position in the frontal plane.

Neural recording and data analysis. Single-unit activity was recorded with miniaturized wireless headstages (Triangle BioSystems International) using the Cerebus data acquisition system (Fan et al., 2011). The chronically implanted electrode array was connected to a wireless transmitter cap $(\sim 3.8 \mathrm{~g})$. Attached to the front end are miniature LEDs (2 $\mathrm{mm}$, Osram, powered by 2 Energizer 379-type batteries). During recording sessions, single units were selected using online sorting. Before data analysis, using Offline Sorter (Plexon), the waveforms were sorted again to eliminate noise and multiunit waveforms (Fan et al., 2011; Rossi et al., 2013a). Only single-unit activity with a clear separation from noise (at least 5 to 1 compared with the noise band) was used for the data analysis (Nicolelis, 2007). The behavioral and electrophysiological data were recorded with a Cerebus data acquisition system and analyzed with MATLAB, Neuroexplorer, and Graphpad Prism. 

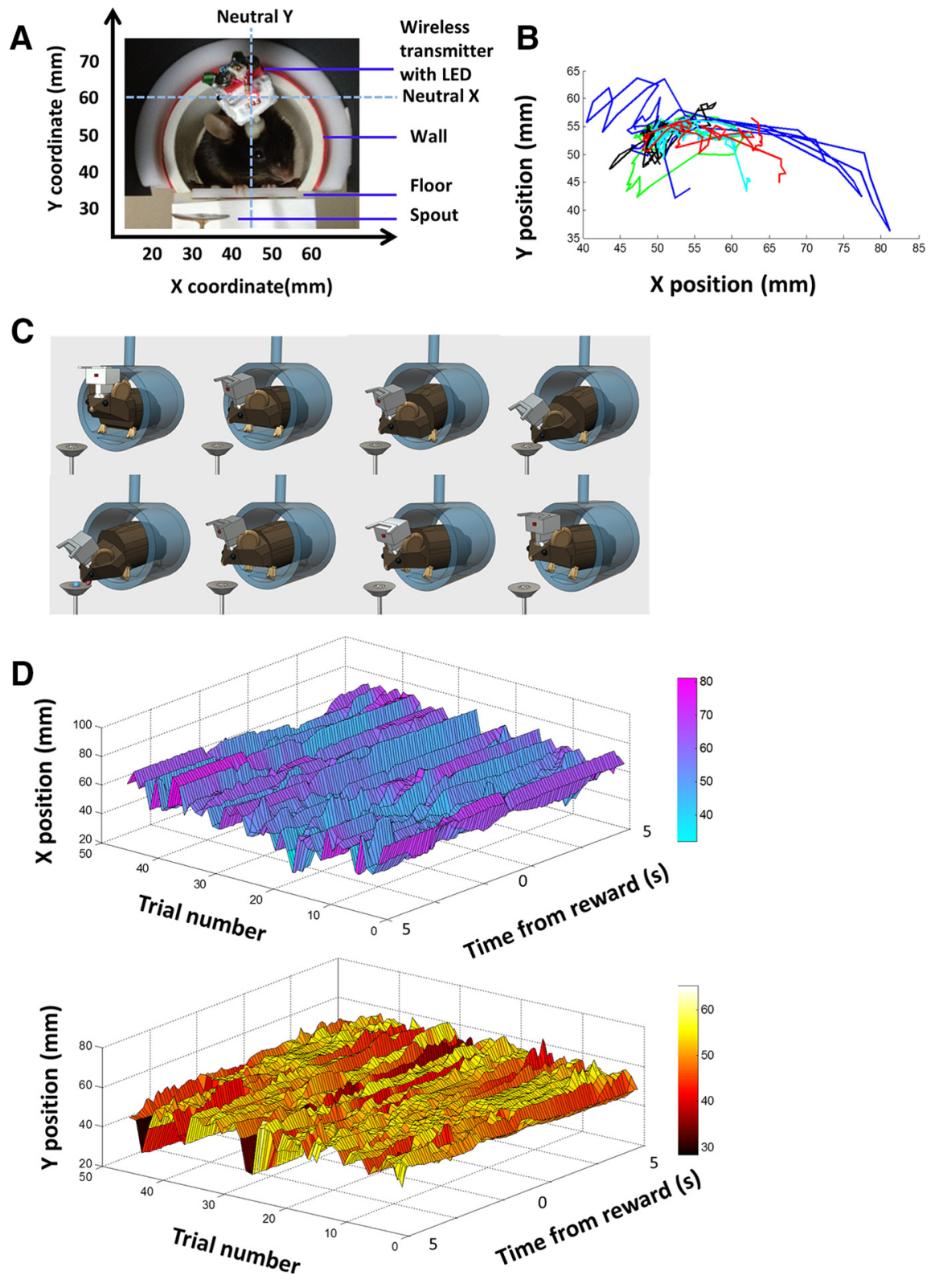

Figure 2. Illustration of behavioral task and wireless recording. $\boldsymbol{A}$, Illustration of in vivo wireless multielectrode recording and the behavioral task. The mouse perches on a small platform, where it is free to move, but locomotion is not possible as the platform is elevated. On its head is a miniaturized 16-channel wireless headstage $(1.5 \times 1 \times 1.5 \mathrm{~cm})$ weighing $\sim 3.8 \mathrm{~g}$ and connected to a chronically implanted multielectrode array targeting the SNr. Sucrose solution is delivered into the spout periodically, preceded by a brief tone. Position of the LED is defined on a Cartesian plot with the ordered pair $(x, y)$. $\boldsymbol{B}$, Illustration of variability in movement trajectory. Each color represents position change during a single trial. C, Diagram illustration of the movement to collect sucrose from the spout. $\boldsymbol{D}$, Representative illustration of LED position from 45 consecutive trials in a session. The $x$ - and $y$-coordinates are plotted separately.

\section{Results}

\section{Histology and single-unit classification of putative}

GABAergic neurons

Using chronically implanted 16-electrode arrays, we recorded from putative GABAergic output neurons in the $\mathrm{SNr}$ (Fig. 1). The nissl-stained brain slices showed that the electrode arrays in all mice were placed in the medial $\mathrm{SNr}$, which receives projections primarily from the dorsal striatum (Bolam et al., 1993; Parent and Hazrati, 1995).

The putative GABAergic output neurons, which constitute the majority of neurons in the SNr, are known to inhibit downstream structures (Chevalier et al., 1984; Gerfen and Wilson, head position (Fig. 3).
1996; Hikosaka, 2007; Zhou and Lee, 2011). They are characterized by high firing rates and narrow spike waveforms (Hikosaka, 2007; Jin and Costa, 2010; Zhou and Lee, 2011; Fan et al., 2012; Rossi et al., 2013a; Barter et al., 2014). For cell type classification, we used a criterion of $160 \mu$ s for the FWHM. As shown in Figure $1 E$, the FWHM of all neurons classified as putative GABAergic output neurons is $<160 \mu \mathrm{s}$.

\section{Behavior}

We tracked the position of the LED on the head of the mouse at 30 frames/s. From the video recording, we were able to obtain the Cartesian coordinates of the head position (Fig. 2A). Because our camera is facing the animal, the Cartesian coordinates reflect the observer's view of the mouse. An increase in the $x$ value indicates movement to the left of the mouse, whereas a decrease indicates movement to the right. An increase in the $y$ value indicates upward movement, whereas a decrease indicates downward movement.

All mice reliably moved following the tone to consume sucrose from the spout. Given the use of a single LED for video tracking, we cannot rule out whether the movement included head or eye rotation, in which case Listing's law becomes relevant. Unlike primates, however, rodents rarely make eye or head rotations independently of the rest of the body. The primary movements we measured are not restricted to only head rotations but involved the entire body. A typical movement is illustrated in Figure 2C. Even though there is no locomotion, such a movement involves changes in multiple joint angles. Each movement involves a unique set of changes in position coordinates (Fig. 2D). Although they may appear to be similar to the observer, the quantification of the position coordinates reveals the variability in the movement kinematics. The variability in movement kinematics in unrestrained animals is in agreement with classic studies of human movements (Bernstein, 1967).

\section{Neural activity reflects position coordinates}

From the motion tracking data, we obtained $x$-and $y$-coordinates of the head LED position at 30 frames/s. When these are compared with the single-unit activity, we observed a striking correlation between the firing rate and the Cartesian coordinates of

The activity of $76 \%$ (243 of 318 ) of all putative GABAergic neurons is significantly correlated with the Cartesian coordinates of head position ( $p<0.001$ for Pearson correlation). Because each movement involves changes in both $x$ - and $y$-coordinates (Fig. 2B), these measures can be correlated with each other. Be- 

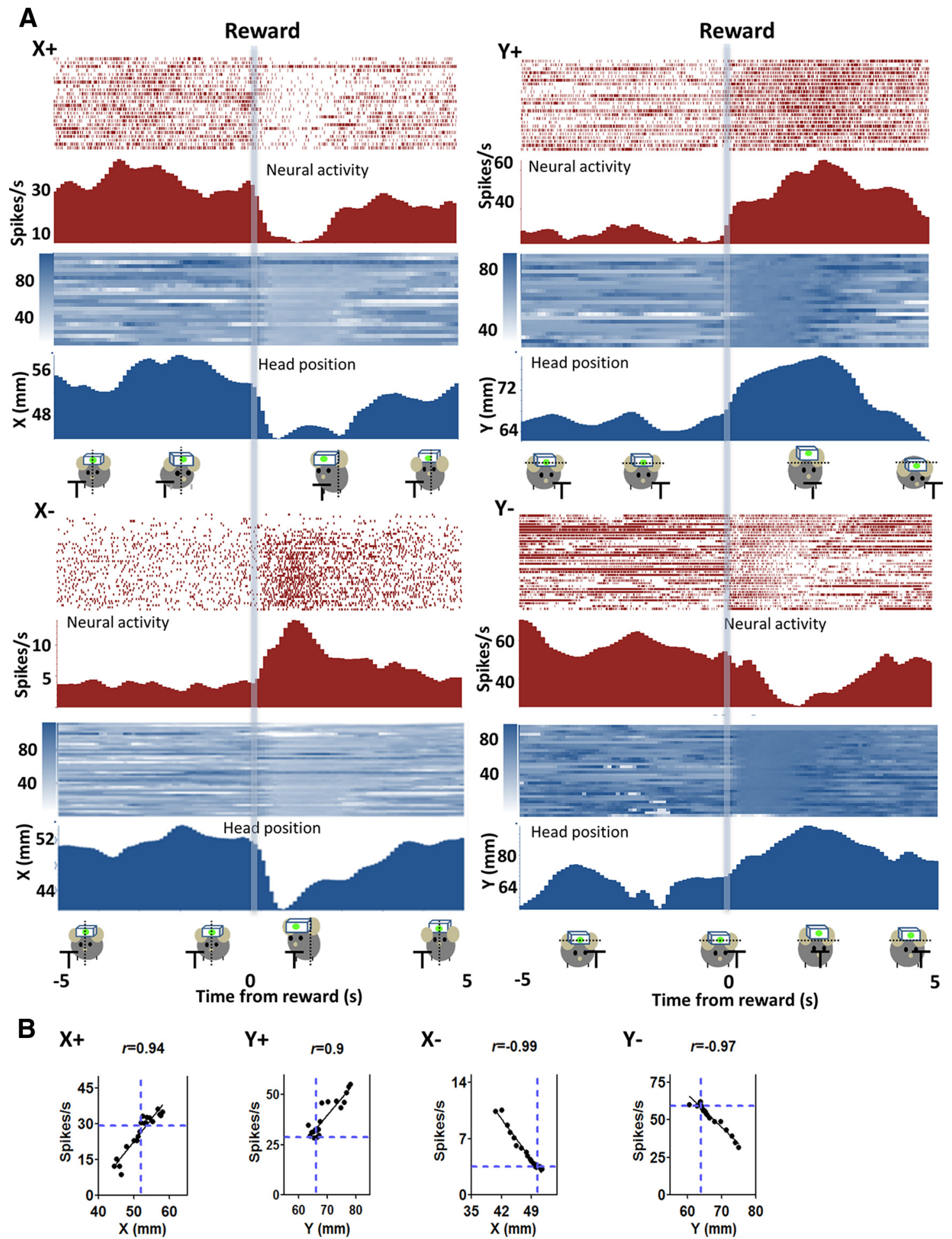

Y- $\quad r=-0.97$

Figure 3. Relationship between single-unit activity from $\mathrm{SNr}$ and raw position coordinates during movement. $\boldsymbol{A}$, The firing rate of SNr neurons exhibits high correlation with instantaneous position coordinates. The position is defined from the edge of the frame in the captured image. Higher $x$-coordinate values indicate positions to the left of the resting neutral position, whereas lower $x$ values indicate positions to the right. Raster plot of a neuron showing a positive correlation with $x$-coordinates of the head position as measured by the LED $\left(X^{+}\right.$neuron, $\left.p<0.001\right)$. The mouse initiates a movement, lowering its head and moving slightly to reach the spout, where sucrose solution $(10 \%, 13 \mu l)$ is delivered $\sim 2$ s later (transparent blue bar). $Y^{+}$neuron, Positive correlation with $y$-coordinates $(p<0.001) ; X^{-}$neuron, negative correlation with $x$-coordinates $(p<0.001) ; Y^{-}$neuron, negative correlation with $y$-coordinates $(p<0.001)$. Relative changes in firing rate and position are shown on the right $y$-axis. $B$, Correlation between firing rate and instantaneous position coordinates.

cause the movements are not perfectly diagonal, however, $x$ - and $y$-coordinates do not change equally (Fig. $2 B$ ). To distinguish between them, we classified a neuron as being correlated with $x$ or $y$-coordinates only when the correlation is very high $(p<$ 0.001 ). We were able to identify neurons with activity reflecting either the $x$ coordinates or the $y$-coordinates of the instantaneous position.

For movement in any direction, single-unit activity was either positively or negatively correlated with the change in position signals (Fig. 3). Among the $x$-correlated neurons, one class in- 
A

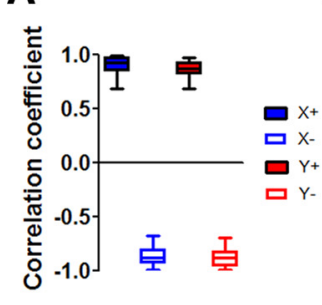

B

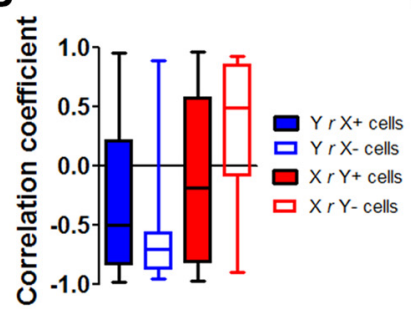

C

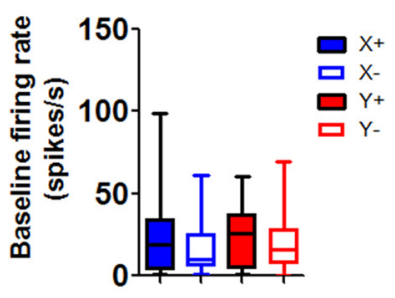

D

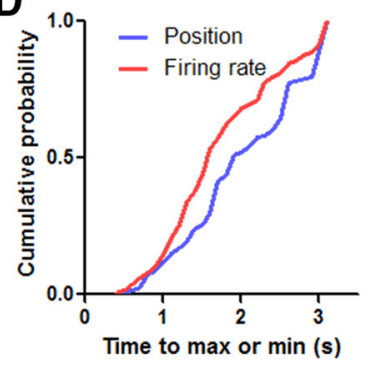

Figure 4. Summary of different types of nigral neurons. $A, r$ values for the four types of neurons identified, showing high correlation between firing rate and either $x$ - or $y$-coordinate. $B, r$ values for the four types of neurons identified, showing much reduced correlation between firing rate and the alternative coordinate. For example, activity of $X^{+}$neurons is poorly correlated with the $y$-coordinate. C, Baseline firing rate during a $2 \mathrm{~s}$ period before trial onset. $\boldsymbol{D}$, Timing of the neural activity (all four classes of neurons) in relation to movement. Cumulative probability distribution of the time it takes from reward delivery and the maximum or minimum value of the firing rates or position coordinates. For the position data, this is approximately the time it takes for the mouse to reach the farthest point from its resting position in either the $x$ - or $y$-axis.

A
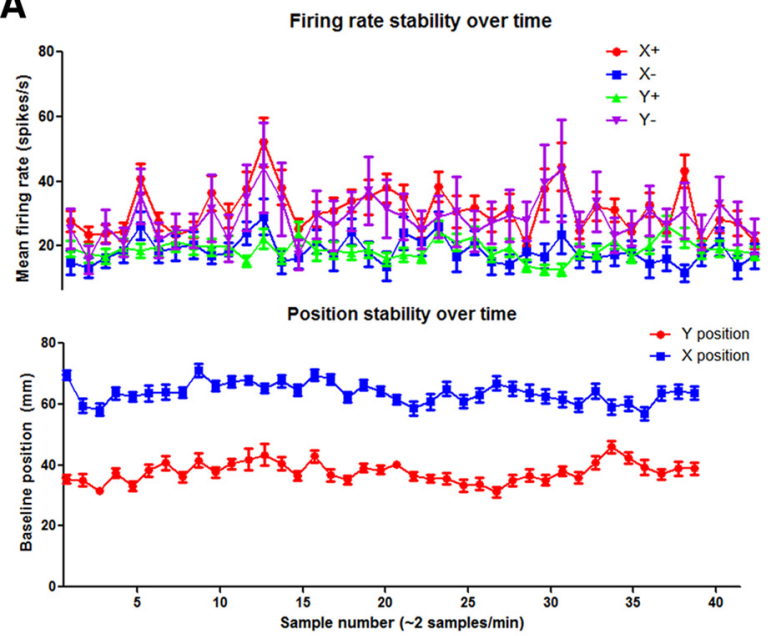

B

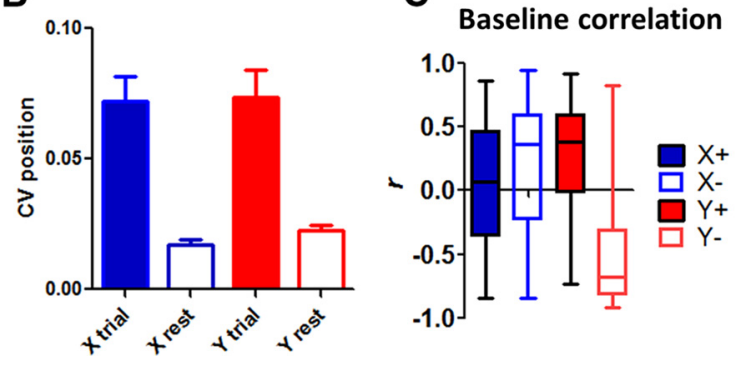

Figure 5. Summary of baseline neural activity and movement. $\boldsymbol{A}$, Stability of position measure and firing rate. We extracted $10 \mathrm{~s}$ time windows during rest periods from all sessions. The position signal and the neural activity are shown. There is no systematic change in these measures during the rest period over the course of a session. $\boldsymbol{B}$, Coefficient of variation (CV, SD/mean) in the position measure during a trial and during the rest period. During the rest period between trials, there is little movement as indicated by much lower CV values in the position. C, Correlation between firing rate and during the rest period, when there is little movement. creases firing when the animal moves to its left side $\left(\mathrm{X}^{+}\right)$and decreases firing rate when moving to the right. The firing rate is positively correlated with the $x$-coordinates of the LED position $(n=$ 100 , mean firing rate $=23.0 \pm 2.1 \mathrm{~Hz}$, mean $r=0.91 \pm 0.008, p<0.001$ ) but poorly correlated with $y$-coordinates (mean $r=-0.29 \pm 0.05$ ). Another class shows the opposite pattern, increasing firing rate when the animal moves to the right (" $\mathrm{X}{ }^{-}$neurons," negatively correlated with $x$-coordinates $(n=39$, mean firing rate $=17.5 \pm 2.6 \mathrm{~Hz}$, mean $r=$ $-0.84 \pm 0.01, p<0.001)$, but less correlated with $y$-coordinates (mean $r=$ $-0.61 \pm 0.05)$.

Among the $y$-correlated neurons, " $\mathrm{Y}^{+}$ neurons" are positively correlated with the $y$-coordinates: they increase firing with upward movement $(n=21$, mean firing rate $=24.4 \pm 4.1 \mathrm{~Hz}$, mean $r=$ $-0.85 \pm 0.02, p<0.001)$, but poorly correlated with $x$-coordinates (mean $r=$ $0.33 \pm 0.12)$. " $\mathrm{Y}^{-}$neurons" $\left(\right.$Down $\left.^{+}\right)$are negatively correlated with the $y$-coordinates: they increase firing with downward movement $(n=83$, mean firing rate $=20.8 \pm 2.0 \mathrm{~Hz}$, mean $r=0.87 \pm 0.009, p<0.001)$, but poorly correlated with $x$-coordinates (mean $r=-0.12 \pm 0.07$ ). Figure 4 show the correlation coefficient ( $r$ values) for all four types of neurons. Neurons that are correlated with $x$-coordinates are poorly correlated with the $y$-coordinates, and vice versa. On the other hand, these different types of neurons showed similar baseline firing rates.

One question is whether the change in neural activity precedes the change in position. If so, then the neural activity could be a signal used to generate the movement. To assess the relative timing of the change in neural activity and movement, we also measured the time it takes for the firing rate or movement to reach the maximum value or minimum value (depending on the type of neuron) after the reward delivery (Fig. 4D). This is approximately the time it takes for the animal to reach the sucrose spout. We found that the neural activity usually preceded the movements, suggesting that the nigral output is used to generate the change in head position. It should be noted, however, that higher temporal resolution in video tracking will be needed to provide a better estimate of the lag between neural activity and movement.

Another question is whether the observed correlation during movement is still present in the absence of any overt movement. We compared the correlation between neural activity and position during trials and a comparable period between trials (a $10 \mathrm{~s}$ window from the intertrial interval just before the onset of the trial). Not surprisingly, the coefficient of variation in position was much lower during the resting period (Fig. 5), showing less movement during this period. As expected, because there was little variation in either the neural activity or position, the correlation was also much weaker.

\section{Normalization of neural activity}

The overall average firing rates of recorded neurons are highly variable. For all neurons in a particular class, the correlation with the position coordinate is highly similar, despite large variations 
A
Raw

$X+$

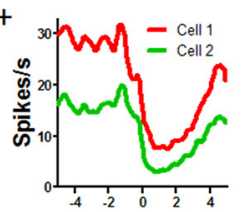

X-

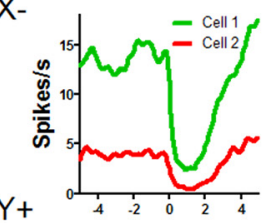

$\mathrm{Y}+$
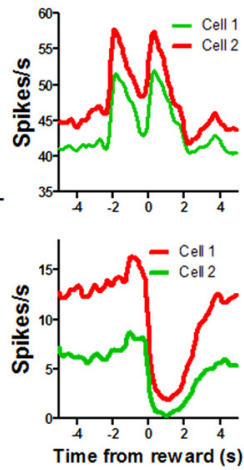

B

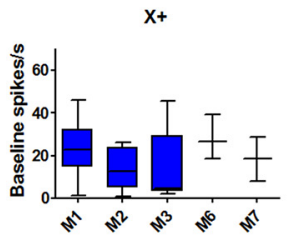

$\mathrm{Y}+$

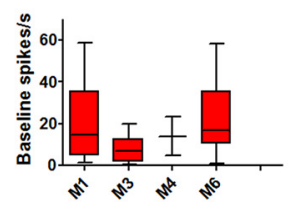

Normalized
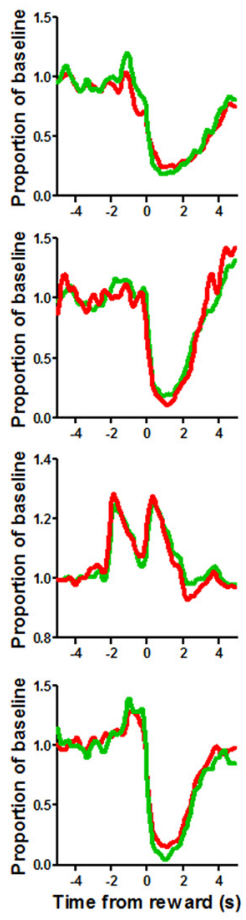

C

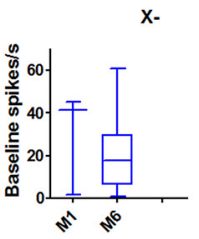

Y.

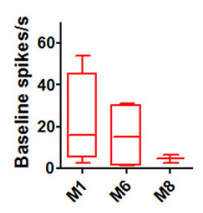

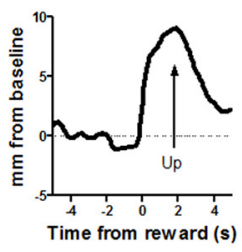

Position
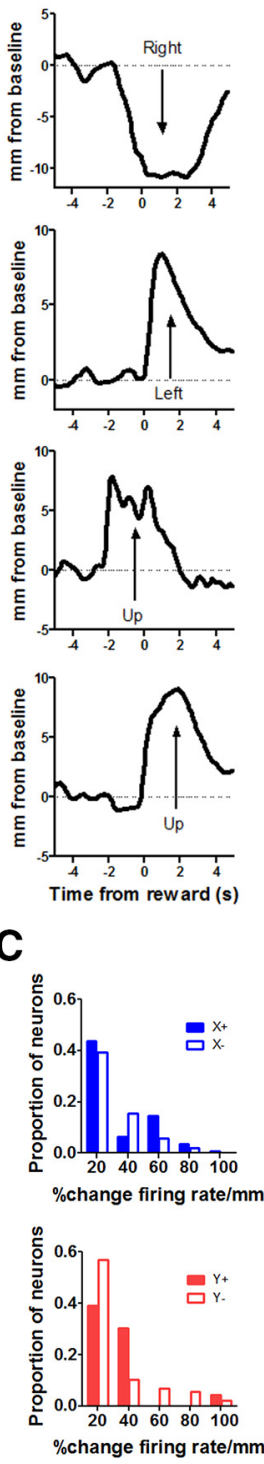

Figure 6. Normalization of firing rates in $\mathrm{SNr}$ neurons. $A$, Each row includes raw firing rates and normalized firing rates from two neurons, and corresponding position change from a different mouse. Left column, Raw firing rates of two neurons of a given type from a mouse. Middle column, Normalized firing rates of the same neurons. The rates are normalized by dividing by the baseline rate of each neuron. Right column, Position change. The relative change in position from baseline is shown, as absolute position values are defined by the edge of the camera frame. $\boldsymbol{B}$, The range of baseline firing rates in neurons from the four different classes. Each graph represents the range of baseline firing rates from a single session. Data from all mice with multiple neurons from a class are shown. There is considerable variability in baseline firing rates, although the correlation with position coordinates is highly similar. C, Distribution of firing rate modulation (\% of baseline) per millimeter. Most neurons show comparable degree of modulation relative to movement amplitude.

in baseline firing rates (Fig. 6). When the firing rate is normalized (divided by the baseline), the neurons show a remarkably uniform response in relation to changes in position coordinates. This is found in all four classes of neurons. Thus, a given change in position coordinates is not accompanied by a certain amount of change in absolute firing rate, but rather in percentage change in firing rate. For example, a neuron that fires at $30 \mathrm{~Hz}$ at the onset of the movement can reduce its firing rate to $15 \mathrm{~Hz}$ during a particular movement, whereas a neighboring neuron that fires at $14 \mathrm{~Hz}$ will reduce its firing rate to $7 \mathrm{~Hz}$. When normalized, both
A

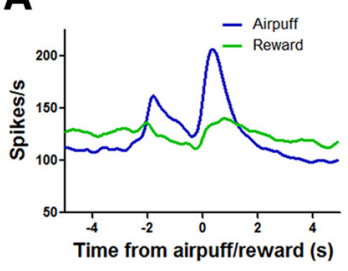

Air puff trials Reward trials
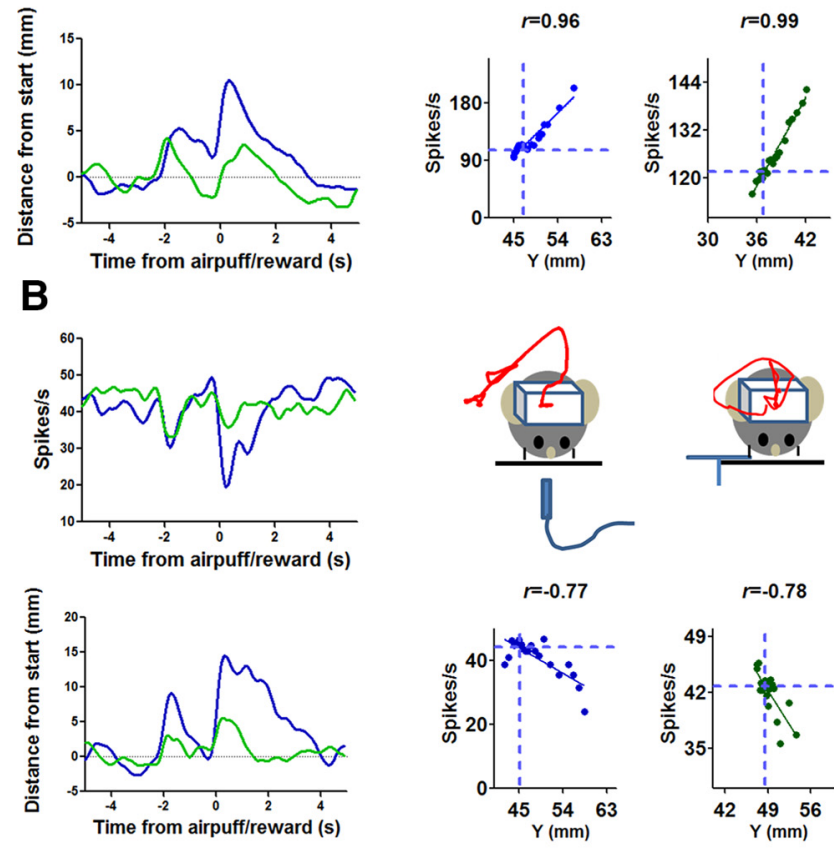

C

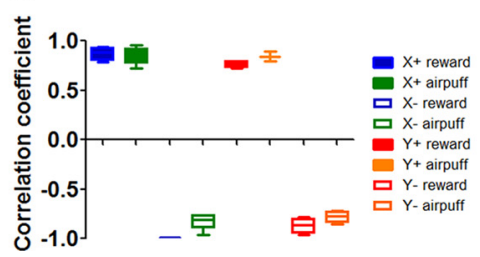

Figure 7. Relationship between firing rate and Cartesian coordinates is similar for both reward and air puff trials. $\boldsymbol{A}$, Left, Firing rate of $\mathrm{Y}^{{ }^{+}}$neuron in relation to air puff/reward. The activity of the same neuron from both types of trials is shown. Right, Example movement trajectory for air puff and reward trials. The correlation between neural activity and Cartesian coordinates is high on both sucrose reward trials and air puff trials. $\boldsymbol{B}$, Firing rate of $\mathrm{Y}^{-}$neuron. Same as above. C, Distribution of $r$ values of all neurons recorded during both reward and air puff trials.

neurons show comparable proportional change from their baseline firing rate. In addition, as shown in Figure $6 C$, the firing rate modulation (\% of baseline) needed to move a certain distance (1 $\mathrm{mm}$ ) is similar for most neurons.

\section{Dissociation from reward expectancy}

The BG and dopaminergic projections that modulate BG activity have long been implicated in reward-guided behavior (Kawagoe et al., 1998; Rossi et al., 2013b). Because our behavioral task used a food reward to get the mice to perform specific movements, one obvious question is whether the observed correlation is specific to reward-guided movements. Because the firing rate mirrored the position of the animal, our results suggest that the $\mathrm{SNr}$ output may could be necessary for all actions, rather than reward-guided actions only. The prediction is that, in the absence of any rewards, or in response to an aversive stimulus, $\mathrm{SNr}$ activity should also be correlated with Cartesian coordinates during movements. To test 


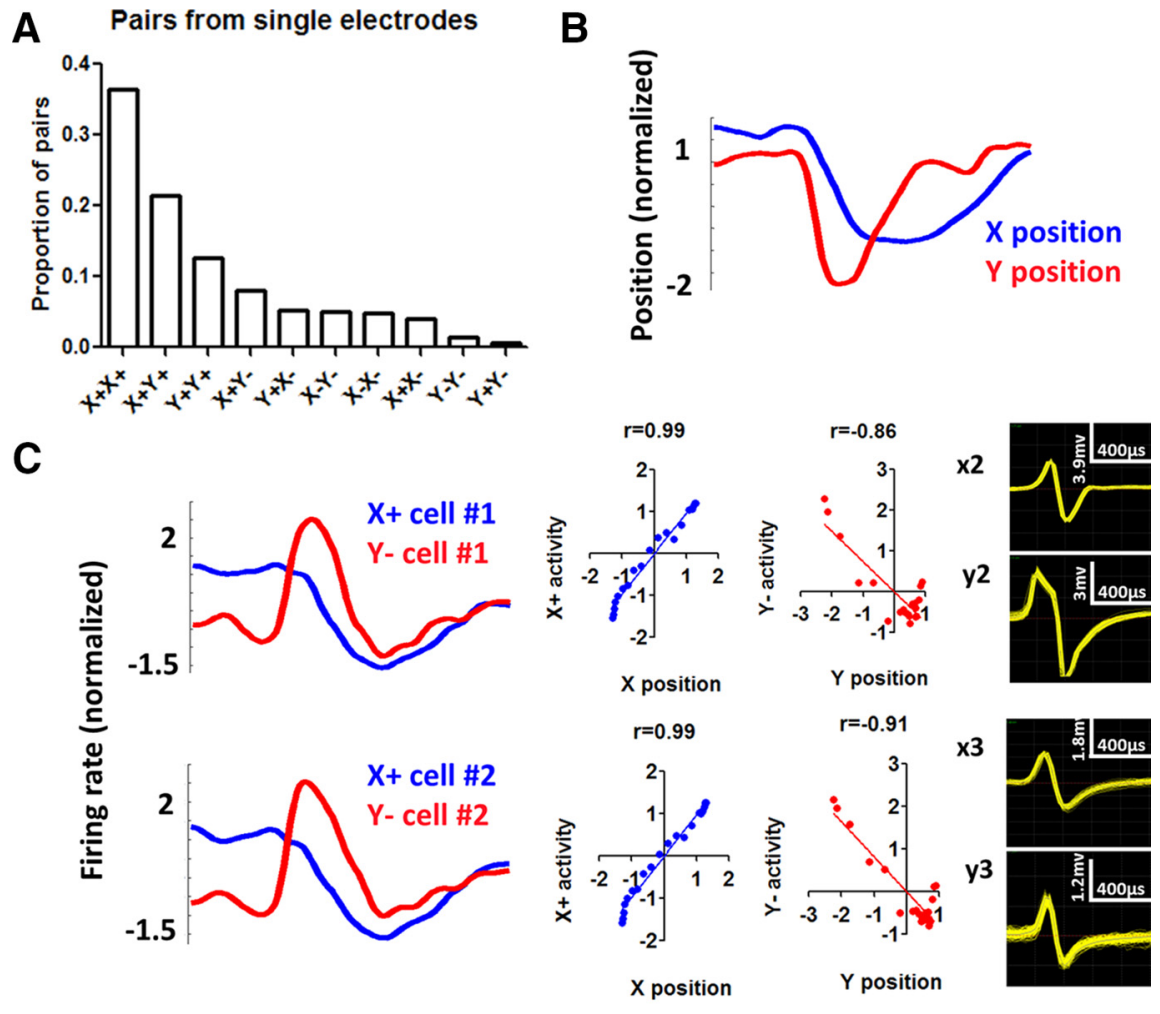

D

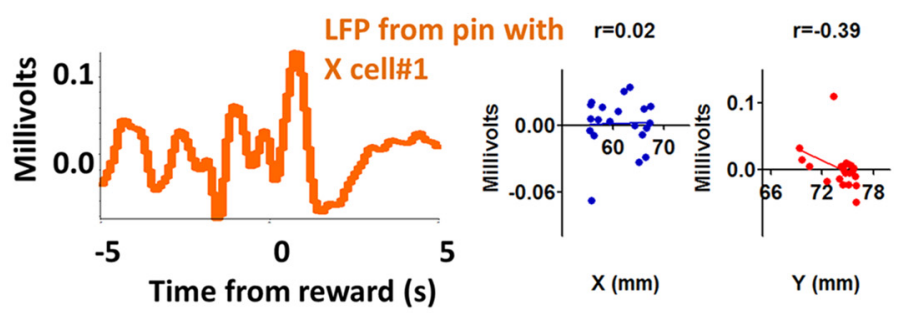

E

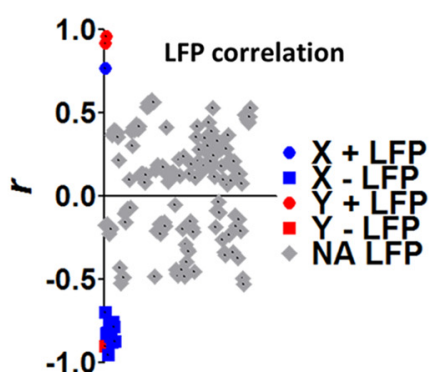

Figure 8. Single-unit activity from a single electrode compared with LFP signal from the same electrode. $\boldsymbol{A}$, Probability of co-occurring neurons recorded from the same electrode. $\boldsymbol{B}$, Illustration of the per-reward change in $x$ - and $y$-coordinates during a single session. $C$, From the same session, $2 X^{+}$neurons and $2 Y^{-}$neurons are shown with their waveforms. All cells show high correlation with position coordinates. Correlation analysis is performed on data from a $10 \mathrm{~s}$ perireward time window $(5 \mathrm{~s}$ before and 5 s after reward delivery). D, LFP recorded from the same electrode as $X^{+}$cell \#1. Correlation between LFP and position coordinates is much weaker. $\boldsymbol{E}$, The distribution of $r$ values for all the recorded LFP channels. Of the 166 LFP channels analyzed, only 15 showed significant correlation with position coordinates (colored, $p<0.001$ ). Thus, when activity is summed from different types of neurons, the resulting correlation between neural activity and position is weaker. The relatively rare examples of LFP being highly correlated with position coordinates suggest that neurons belonging to a particular class (e.g., $\mathrm{X}^{+}$) might be located close to each other.

this possibility, we conducted an additional experiment in 4 mice, in which aversive air puff trials were introduced using the same experimental setup. A block of sucrose trials was followed by a block of air puff trials, or blocks of sucrose and air puff trials alternated in an $\mathrm{ABAB}$ design. On aversive trials, a brief air puff is used as an aversive stimulus that reliably generates avoidance responses (Thompson et al., 1998; Matsumoto and Hikosaka, 2007). We were able to examine the response of the same neurons recorded during sucrose and air puff trials. As shown in Figure 7, on both reward and air puff trials, the mice reliably moved at the time of the cue and at the time of the air puff delivery. The movement trajectory, illustrated by the red trace in the diagram, differed significantly. On air puff trials, instead of approaching the spout, the animal moved away from the air puff. Yet the correlations between position coordinates and neural activity were comparable on both reward and air puff trials. This correlation is therefore independent of reward expectancy. These results suggest that the SNr output is crucial for controlling the kinematics of voluntary movements, regardless of the goal of the movements, whether to seek reward or to avoid harm.

\section{Topographical distribution}

Because each class of neurons classified in the present study is correlated with a unique set of position coordinates, if their firing rates were averaged, the resulting signal would not reflect any movement parameter accurately. We examined the relationship between local field potential (LFP), a measure of population activity in the $\mathrm{SNr}$ recorded using the same electrodes, and position coordinates. As expected, the LFP measure is not highly correlated with position coordinates. Of 166 LFP channels analyzed, only 15 show significant correlation with any of the four position variables (Fig. 8). Thus, more global measures of neural activity, such as LFP or the BOLD signal in AMRI, cannot fully reveal the nigral representation of position coordinates during movement.

An important question is where these different classes of neurons are located in the $\mathrm{SNr}$. Are neurons related to $x$-coordinate and $y$-coordinate position changes located next to each other? Unfortunately, with in vivo recording, it is difficult to ascertain their precise location. To quantify the degree of "intermingling" of recorded neurons, we analyzed all electrodes that recorded activity from more than one neuron. Assuming that a single electrode wire picks up signals from neurons that are located close to each other, the cooccurrence of different neurons on a single electrode can serve as a measure of the degree of intermingling. As shown in Figure 8, it is common for a single electrode wire to detect signals from neurons from different classes (e.g., both $\mathrm{X}^{+}$and $\mathrm{Y}^{+}$neurons). Thus, there appears to be significant intermingling of the different 
classes of neurons, although such a coarse analysis cannot reveal any fine spatial mapping of the neuronal classes. On the other hand, the possibility of topographic distribution of the respective functional cell classes is supported by the high proportions of simultaneous recordings of cells with similar properties, and by the few instances in which LFP was highly correlated with position coordinates (Fig. $8 E)$. Moreover, it is possible that mixed pairs recorded from a single electrode could result from recording in border zones.

\section{Egocentric reference frame and} movement direction

The above results showed that the firing rates of different SNr neurons reflect Cartesian coordinates of the animal's instantaneous head position. But the dynamic range of the neural signal is limited and cannot adequately reflect all position coordinates. The key question is how to interpret the positive and negative correlations between position coordinates captured by the camera and the SNr neural activity.

The Cartesian mapping reflects the observer's view of the animal, as captured by the camera. From the perspective of the mouse that is moving, an egocentric frame of reference is appropriate. If we adopt an egocentric reference frame, then changes in position coordinates reflect movements in four directions: up, down, left, right (Fig. 9). Using the egocentric reference frame, for movement in a particular direction, some neurons increase firing whereas others reduce firing. For example, when the mouse moves to its right, the firing rate of the "right ${ }^{+}$" $\left(\mathrm{X}^{-}\right)$ neurons increases, whereas that of a "left ${ }^{+}$" $\left(\mathrm{X}^{+}\right)$neuron decreases.

Figures 10, 11, 12, and 13 show that the observed correlation between neural activity and instantaneous position coordinates is found for every movement. The same neuron increases firing during movement in its "preferred" direction and reduces firing during movement in the opposite direction.

\section{Discussion}

We found that the firing rate of SNr neurons can reflect Cartesian coordinates of head position during movement. The movements recorded have both horizontal and vertical components, associated with changes in both $x$ - and $y$ - coordinates. The combination of outputs from both $x$ - and $y$-related neurons is sufficient to recreate the trajectory of each movement. Each class of neuron $\left(\mathrm{X}^{+}, \mathrm{X}^{-}, \mathrm{Y}^{+}, \mathrm{Y}^{-}\right)$represents the $x$ or $y$ component of the position vector. The actual position vector is determined by the relative contributions of different classes of neurons.

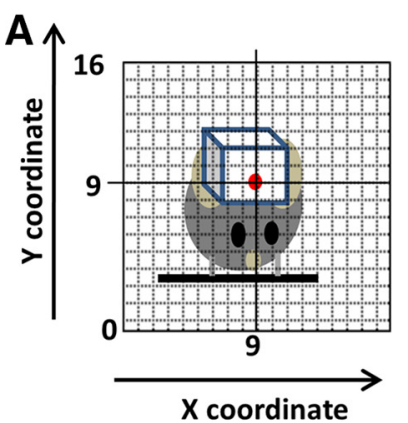

B

Neutral

From origin in video: $(9,9)$

Relative to mouse: $(0,0)$

$\mathrm{X}+/$ Left+ cell: 10 spikes/s

X-/Right+ cell: 20 spikes/s

$\mathrm{Y}+/ \mathrm{Up}+$ cell: 30 spikes/s

Y-/Down+ cell: 15 spikes/s

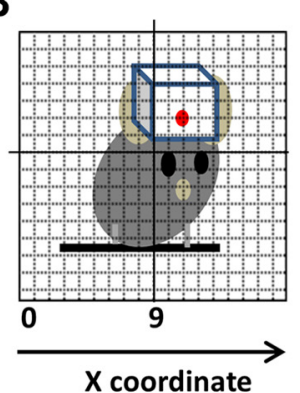

Up-left bending

From origin in video: $(11,11)$

Relative to mouse: $(2,2)$

X+/Left+ cell: 12 spikes/s

X-/Right+ cell: 18 spikes/s

$\mathrm{Y}+/ \mathrm{Up}+$ cell: 32 spikes/s

Y-/Down+ cell: 13 spikes/s

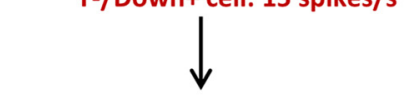

C

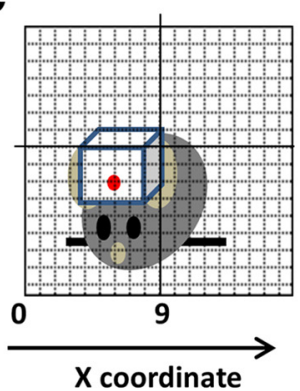

Down-right bending

From origin in video: $(6,7)$

Relative to mouse: $(-3,-2)$

$\mathrm{X}+$ /Left+ cell: 7 spikes/s

$X-/$ Right+ cell: 23 spikes/s

$\mathrm{Y}+/ \mathrm{Up}+$ cell: 28 spikes/s

Y-/Down+ cell: 17 spikes/s
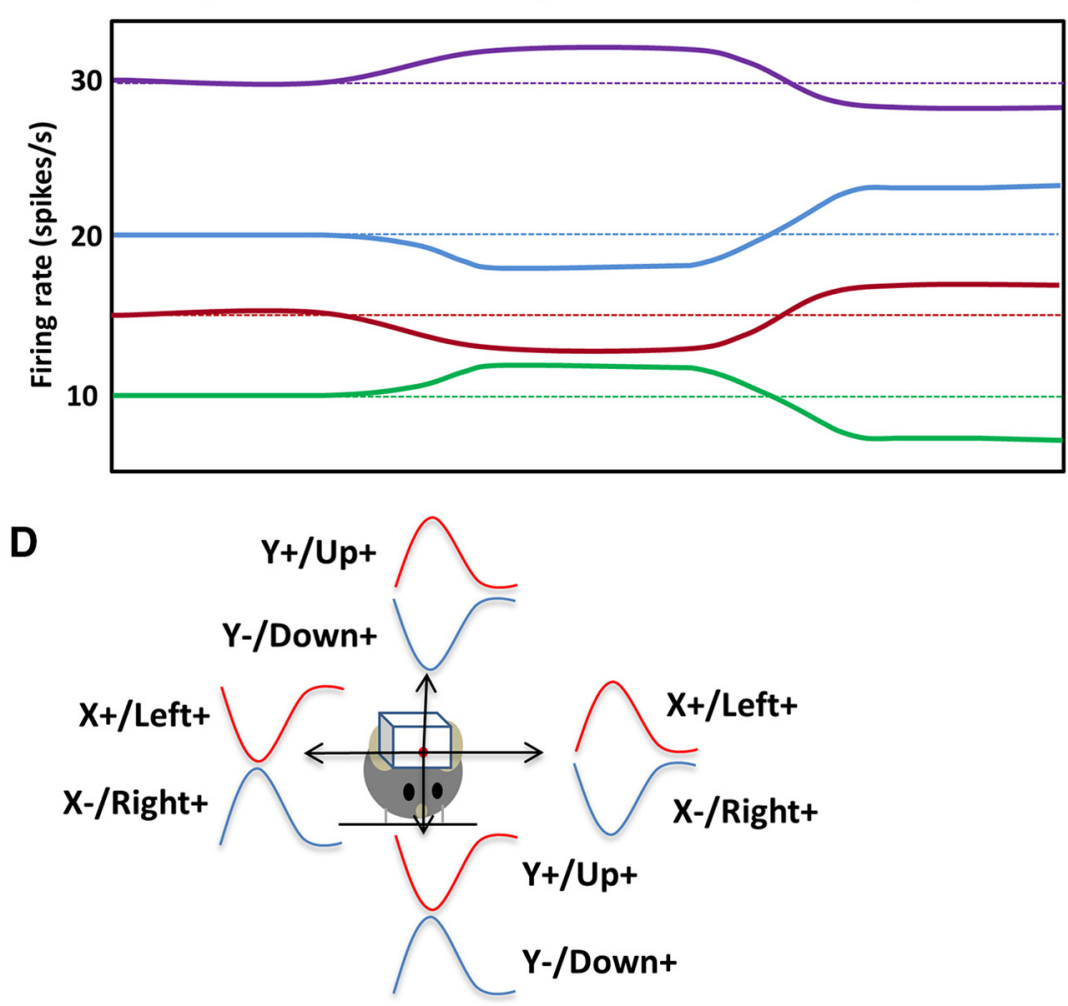

Figure 9. Schematic illustration of different types of neurons. $A$, Illustration of hypothetical mouse movement in relation to the neural activity. The firing rates of the four types of neurons are shown. For this hypothetical example, 1 spike/s is equal to 1 unit of change in $x$ - or $y$-coordinate. The units are arbitrary. The starting position is $(9,9)$. $\boldsymbol{B}$, The mouse moves to its left and up. The LED position changes to $(11,11)$. C, Right, The mouse moves to a new position $(6,7)$. Relative to its start position, the change is $(-3,-2)$. D, Schematic illustration of the relationship between neural activity in relation to movements in four directions. The illustration of neural activity does not represent actual data because we did not record all four types of neurons from a single animal during a session. Opponent activity was observed during movement in any direction. For example, $\mathrm{Y}^{+}\left(\mathrm{Up}^{+}\right)$neurons increase their activity during upward movements and decrease their activity during downward movements. The opposite is true of $\mathrm{Y}^{-}\left(\right.$Down $\left.^{+}\right)$neurons.

We only measured head position, but it is unclear whether similar mechanisms are used for position control of the rest of the body. Moreover, we deliberately restricted $z$-axis movement, but such movement is not completely eliminated. To fully capture the kinematics, 3D motion tracking will be needed. Despite such limitations, our results demonstrate for the first time a continuous and quantitative relationship between BG output and position coordinates during movement. 

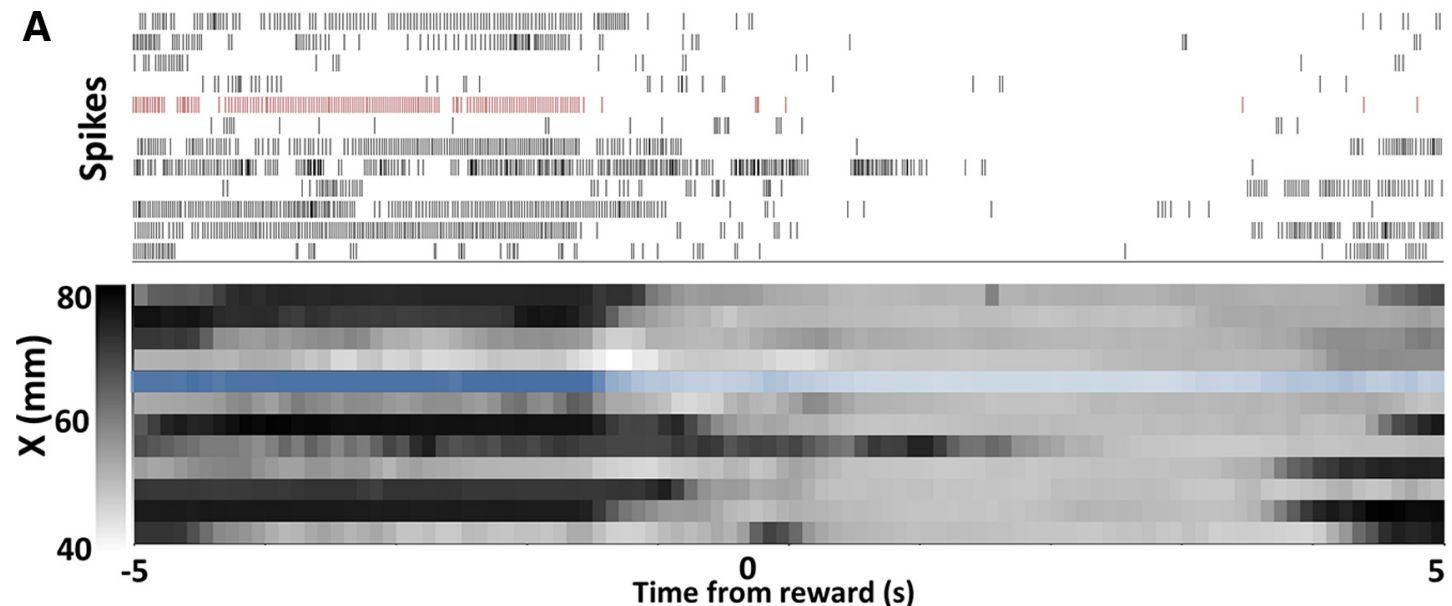

B
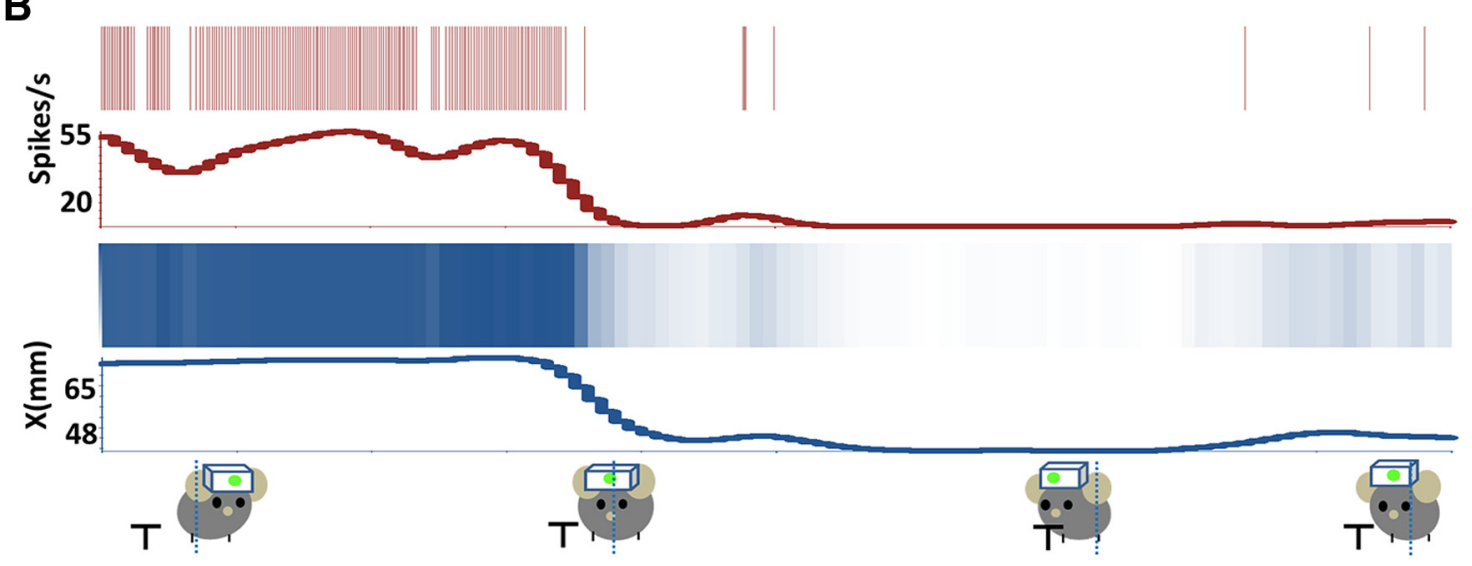

C

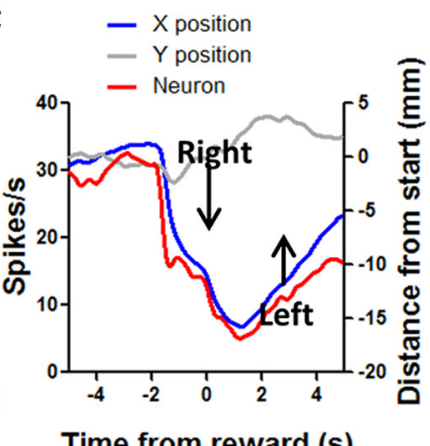

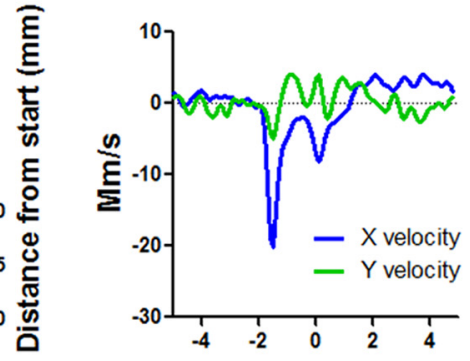

Time from reward (s)

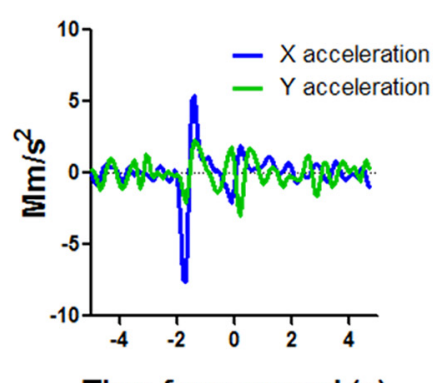

Time from reward (s)

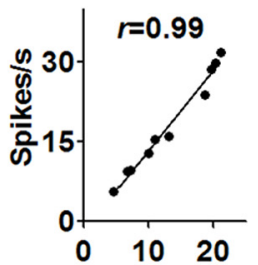

Right distance $(\mathrm{mm})$
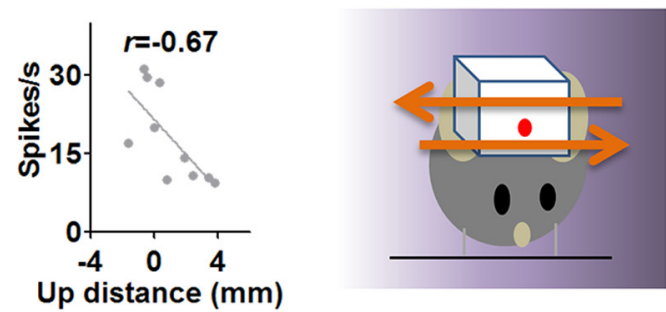

Max firing rate

Min firing rate

Figure 10. Left ${ }^{+}\left(\mathrm{X}^{+}\right)$neuron increases firing during leftward movement and decreases firing during rightward movement. $A$, Detailed illustration of the correlation between firing rate and $x$-coordinates on a trial-by-trial basis. Neural activity and $x$-coordinates from 10 consecutive trials are shown. Each row represents a single trial. $\boldsymbol{B}$, Neural and position data from a single trial are selected and compared. $x$-axis position changes during each movement are reflected in the firing rate of this neuron. The mouse moves to the right to consume sucrose. The activity of the neuron decreased during the rightward movement and then increased when the mouse moves left again to recover its initial starting position. $C$, Average firing rate changes and changes in different movement parameters. In this example, the $y$-component of the movement is much smaller. The plots on the right show velocity and acceleration data for the same movements. $\boldsymbol{D}$, Using an egocentric reference frame, the direction of firing rate changes corresponds to the direction of movement. There was a positive correlation between neural activity and position change in a leftward direction $(r=0.99, p<0.001)$. The correlation with the nonpreferred Cartesian axis is also shown. The diagram on the right shows the gradient of firing rate in relation to position coordinates. 


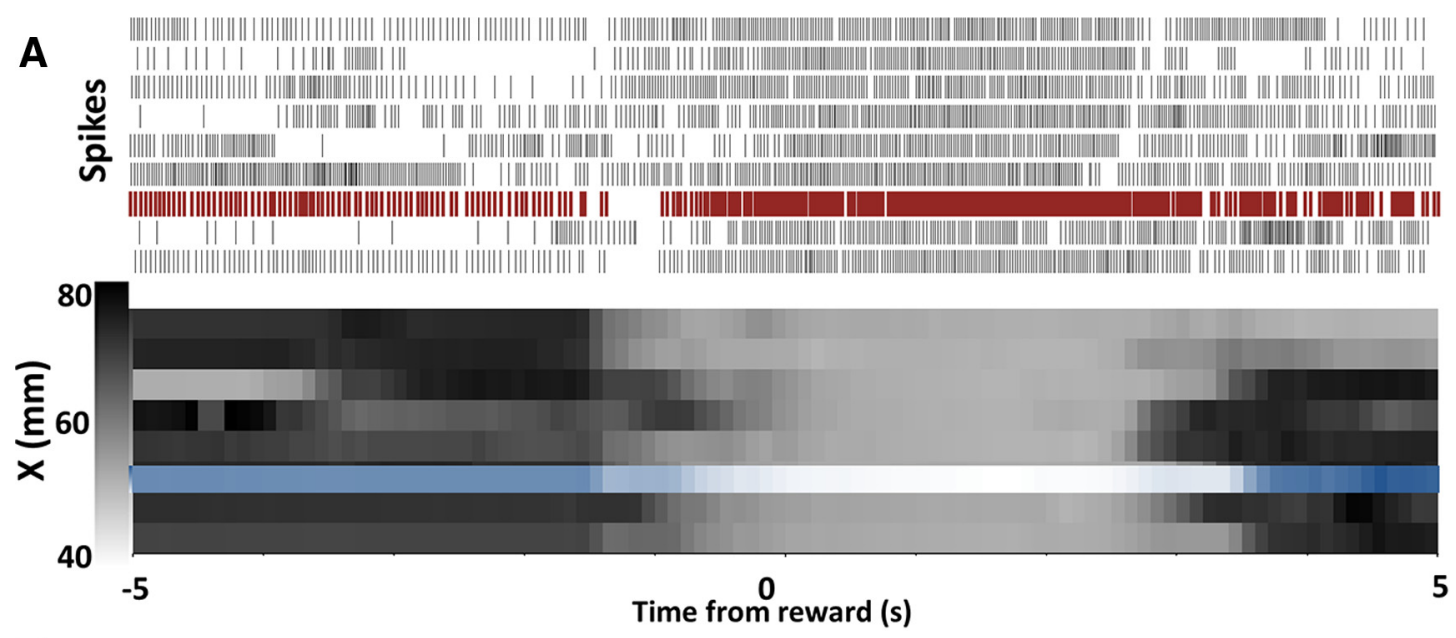

B
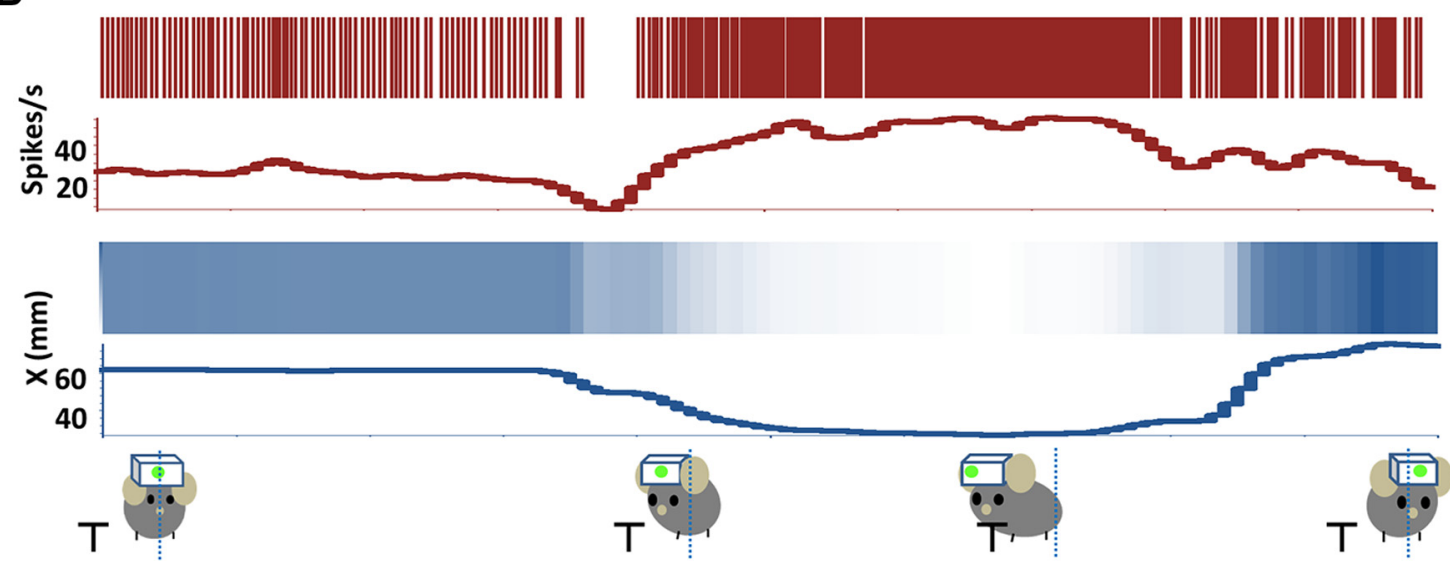

C $\quad-X$ position

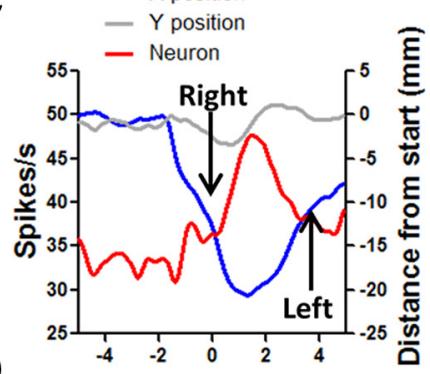

Time from reward (s)

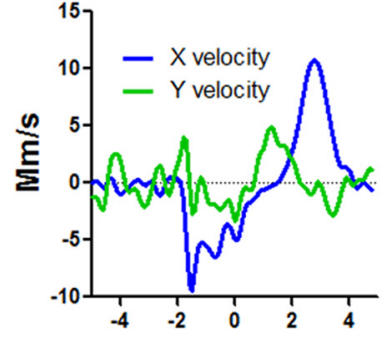

Time from reward (s)

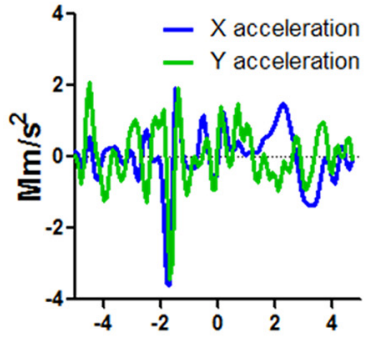

Time from reward (s)
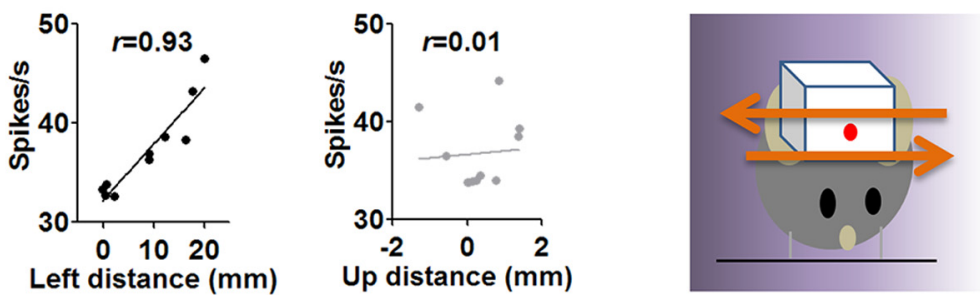

Max firing rate

Figure 11. Right ${ }^{+}\left(X^{-}\right)$neuron increases firing during rightward movement and decreases firing during leftward movement. $A$, Detailed illustration of the correlation between firing rate and $x$-coordinates on a trial-by-trial basis. Neural activity and $x$-coordinates from 10 consecutive trials are shown. Each row represents a single trial. $B$, Neural and position data from a single trial are selected and compared. $x$-axis position changes during each movement are reflected in the firing rate of this neuron. $\boldsymbol{C}$, Average firing rate changes and changes in different movement parameters. The plots on the right show velocity and acceleration data for the same movements. $\boldsymbol{D}$, Using an egocentric reference frame, the direction of firing rate changes corresponds to the direction of movement. There was therefore a negative correlation between firing rate and raw $x$-coordinate value but a positive correlation between firing rate and distance in the rightward direction $(p<0.001)$. The correlation with the nonpreferred Cartesian axis is also shown. The diagram on the right shows the gradient of firing rate in relation to position coordinates. 


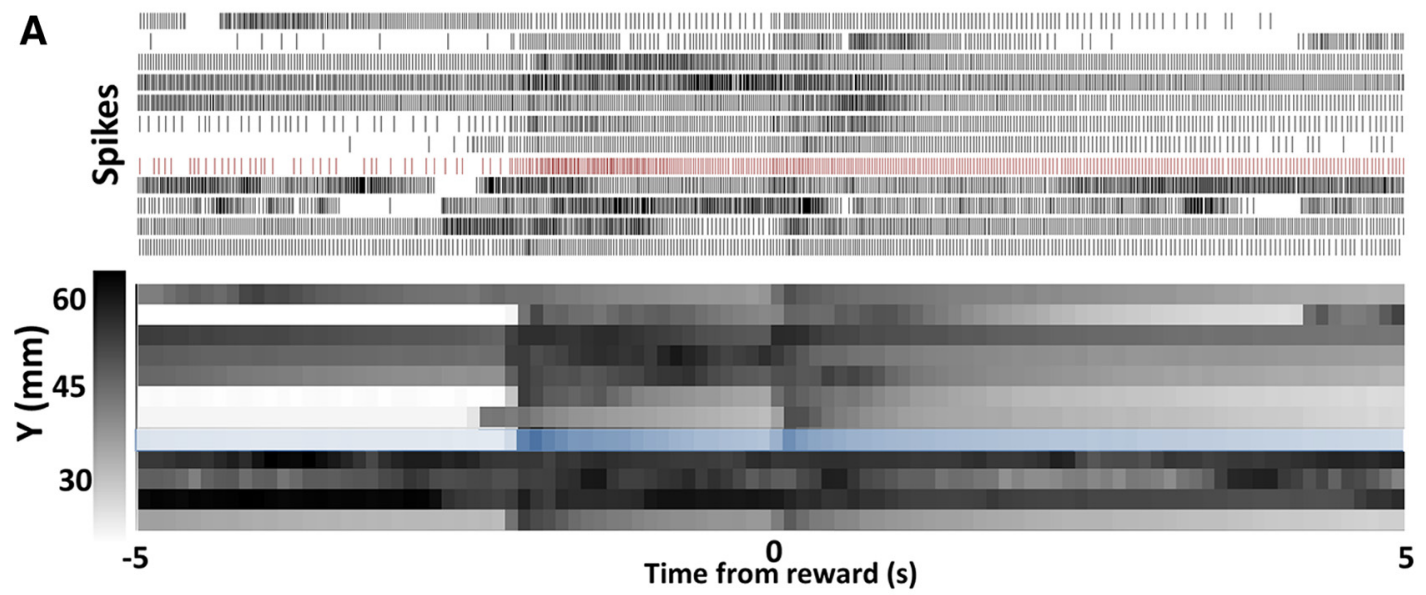

B
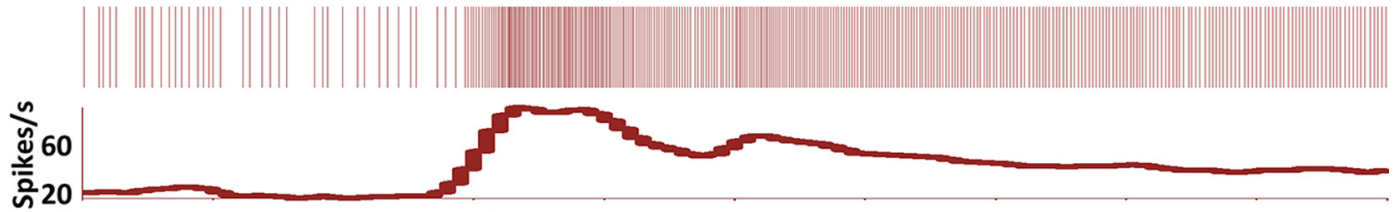

C

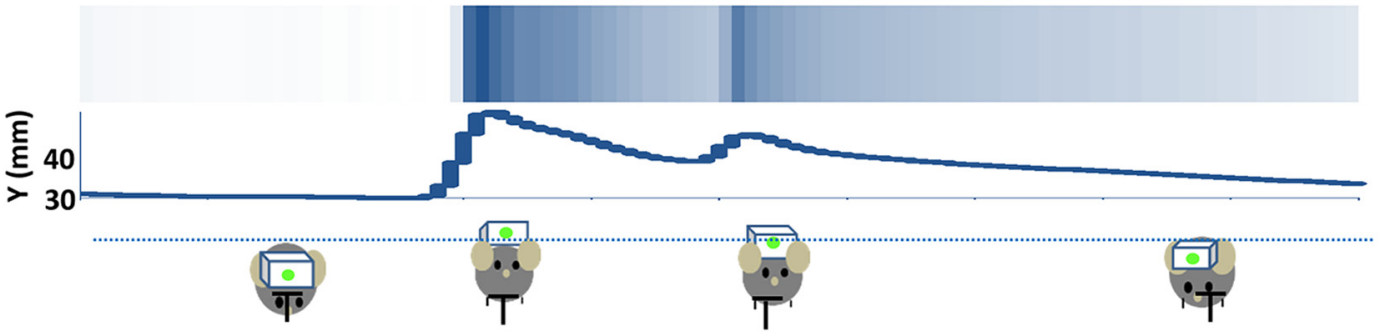

C $\quad-x$ position

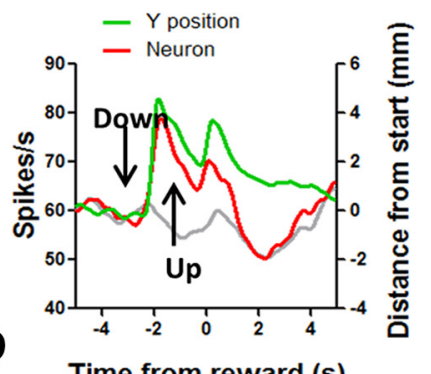

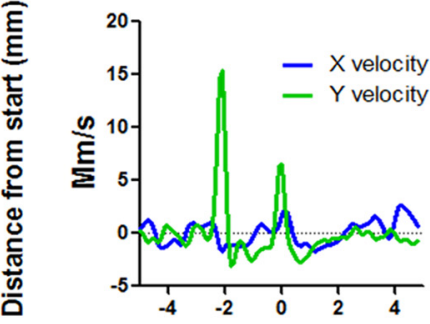

Time from reward (s)

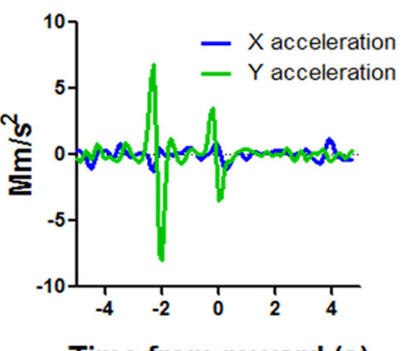

Time from reward (s)

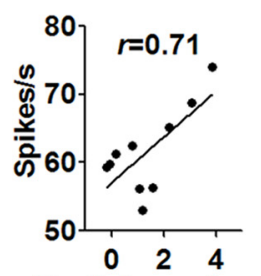

Up distance $(\mathrm{mm})$
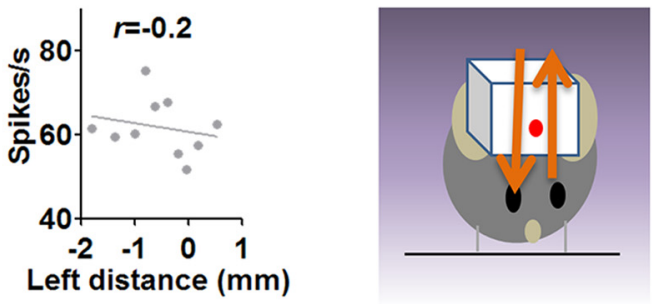

Max firing rate

Figure 12. $U \mathrm{Up}^{+}\left(\mathrm{Y}^{+}\right)$neuron increases firing during upward movement and decreases firing during downward movement. $A$, Detailed illustration of the correlation between firing rate and $y$-coordinates on a trial-by-trial basis. Neural activity and $y$-coordinates from 10 consecutive trials are shown. Each row represents a single trial. $\boldsymbol{B}$, Neural and position data from a single trial are selected and compared. $\boldsymbol{C}$, Average firing rate changes and changes in different movement parameters. The plots on the right show velocity and acceleration data for the same movements. $\boldsymbol{D}$, There is a positive correlation between firing rate and $y$-coordinates and distance in the upward direction $(p<0.001)$. Using an egocentric reference frame, the direction of firing rate changes corresponds to the direction of movement. The correlation with the nonpreferred Cartesian axis is also shown. The diagram on the right shows the gradient of firing rate in relation to position coordinates.

Problems with current models of BG function

According to current models, the BG output tonically inhibits downstream structures, such as tectum and thalamus, when the animal is not moving but transiently disinhibits them to initiate movement (Hikosaka and Wurtz, 1983; Chevalier and Deniau, 1990; Hikosaka, 2007). Such models make no attempt to explain the direction, amplitude, and velocity of movements. Although we frequently found a reduction in firing rate during movement, 
this reduction cannot be equated with disinhibition of downstream structures and behavior. A nigral neuron may reduce its firing rate during leftward movement, but the same neuron increases its firing rate during rightward movement. A change in firing rate therefore reflects a position change in a specific direction, in accord with previous observations of direction tuning in BG neurons (Mitchell et al., 1987; Crutcher and Alexander, 1990).

Moreover, the BG output is not monolithic but consists of at least four types of signals (Fig. 6). For movement along each axis $(x$ or $y$ ), a pair of opponent signals is needed: for example, for leftward movement, the left ${ }^{+}$neuron increases firing as the right ${ }^{+}$neuron decreases firing, and vice versa for rightward movement. In spinal circuits, opponent signals are needed to increase firing of motor neurons innervating a particular muscle and inhibit motor neurons for the antagonistic muscle. But opponent signals from the BG do not reach spinal motor neurons directly. Rather, they control more global variables, such as body configurations, by influencing multiple lower systems (e.g., reticulospinal) controlling joint angle and muscle length, thereby ultimately contracting and relaxing many muscles (Yin, 2014a).

Previous models assume that the basal ganglia are important for action selection by inhibiting unwanted action and disinhibiting desired action. What the "action" consists of is never defined, and what exactly is inhibited or disinhibited by the BG is never explained. Usually, what is meant is some overt behavioral output, which is assumed to be all or none. Consequently, although opponent BG outputs were recognized in earlier work, their functional significance was interpreted differently. According to the "focused selection" model (Mink, 1996), a pause in some BG output disinhibits an action, such as reaching; but because only one action can be performed at a time, there must be a simultaneous increase in other BG outputs, to inhibit competing motor programs, such as postural fixation of the arm (i.e., not reaching). The assumption is that posture and movement are antagonistic, and that, to move, it is necessary to turn off posture control. By contrast, our results suggest that there is no antagonism between posture control and movement, movement being simply a change in posture. Rather, the antagonism exists between systems that move the body in opposite directions. It is therefore necessary to abandon the biased perspective of the naive observer, from which behavior appears to be a series of discrete events. Instead, behavior must be studied as a continuous process, and neural activity as continuously varying.

D
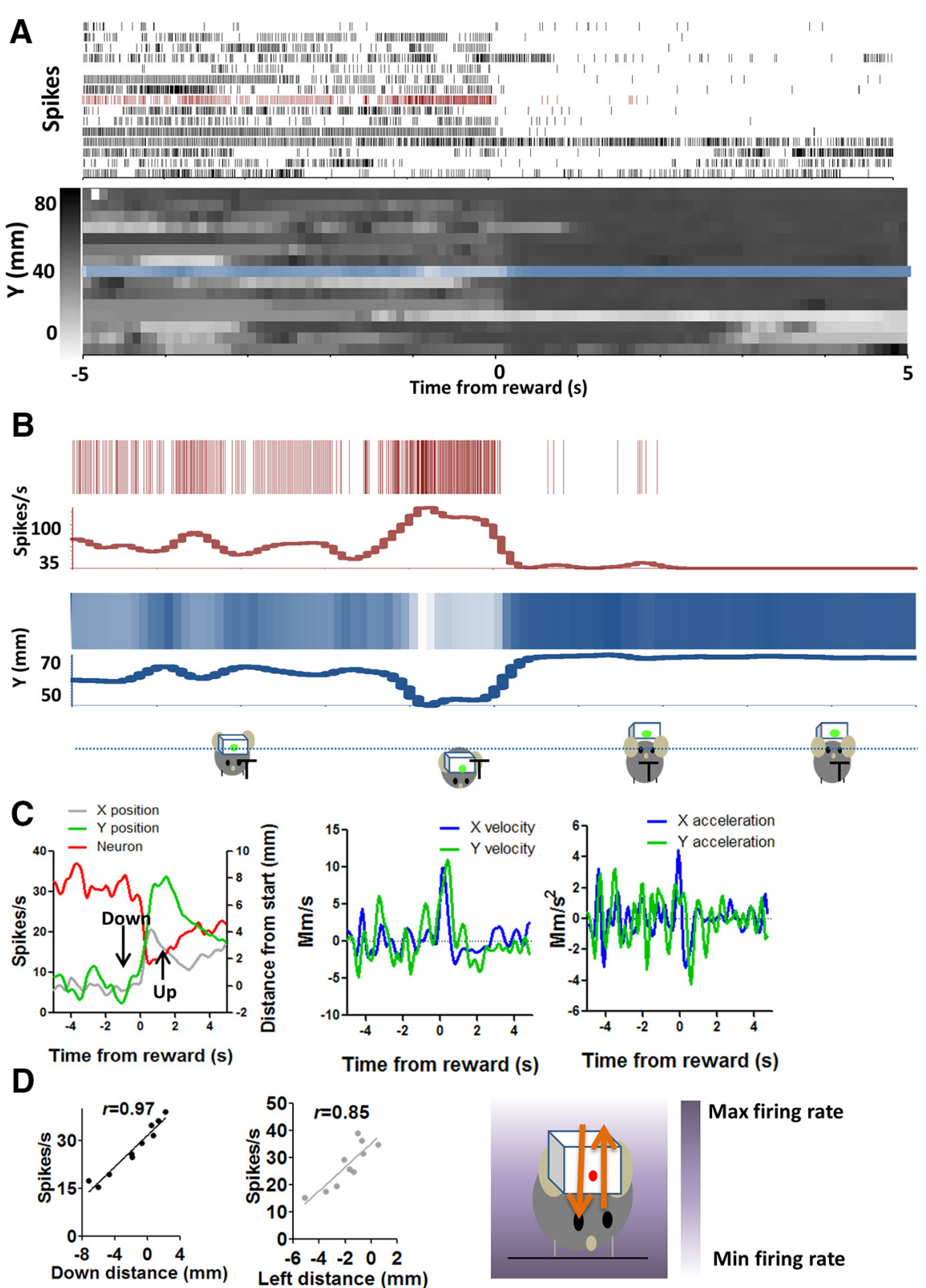

Figure 13. Down ${ }^{+}\left(\mathrm{Y}^{-}\right)$neuron increases firing during downward movement and decreases firing during upward movement. $A$, Detailed illustration of the correlation between firing rate and $y$-coordinates on a trial-by-trial basis. Neural activity and $y$-coordinates from 10 consecutive trials are shown. Each row represents a single trial. $\boldsymbol{B}$, Neural and position data from a single trial are selected and compared. $\boldsymbol{C}$, Average firing rate changes and changes in different movement parameters. The plots on the right show velocity and acceleration data for the same movements. $\boldsymbol{D}$, Using an egocentric reference frame, the direction of firing rate changes corresponds to the direction of movement $(p<0.001)$. There was therefore a negative correlation between firing rate and raw $y$-coordinate value but a positive correlation between firing rate and distance in the downward direction $(p<0.001)$. The correlation with the nonpreferred Cartesian axis is also shown. In this case, the correlation with distance traveled in the leftward direction is also high because the movement is nearly diagonal, so that the $x$ and $y$ position changes are similar. However, the correlation with y is still considerably higher, and trial-by-trial examination of the data confirms that this neuron is selective for the $y$ component of the position vector. The diagram on the right shows the gradient of firing rate in relation to position coordinates.

Our results are also at odds with the so-called rate model (DeLong, 1990), according to which the direct (striatonigral) and indirect (striatopallidal) pathways converge on the same $\mathrm{SNr}$ (and internal globus pallidus) output neurons, one decreasing while the other increasing the monolithic BG output, which determines the degree of inhibition of movements. The different BG pathways can thus "grade" the amplitude and speed of movements. Although this model attempts to explain movement kinematics, it incorrectly assumes that both movement amplitude 


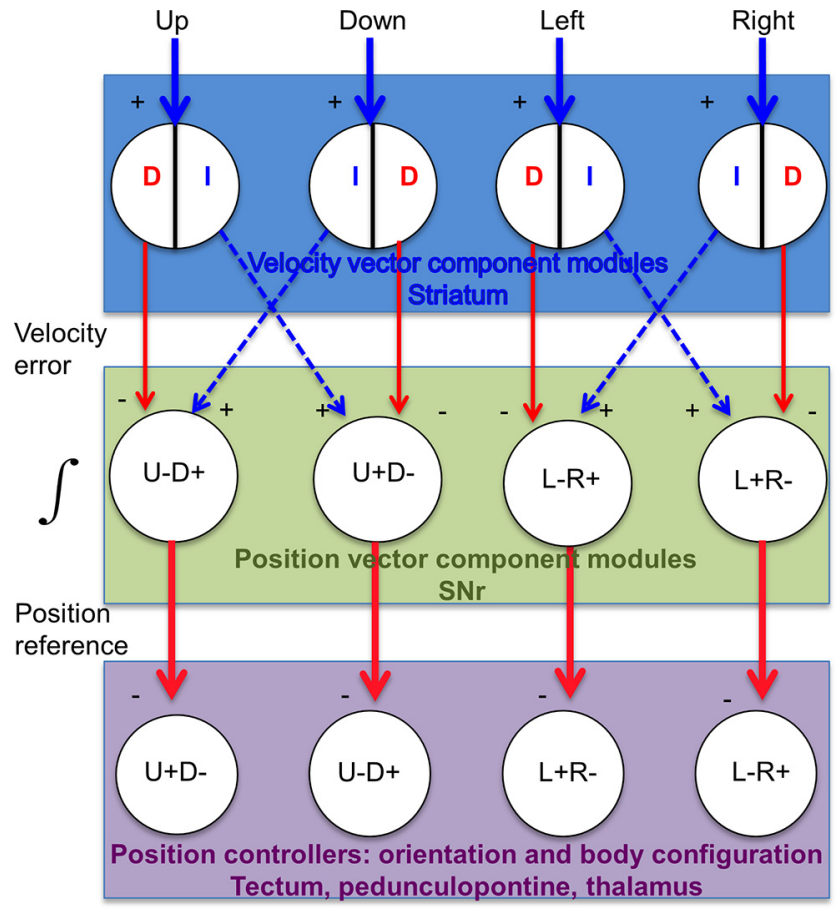

Figure 14. Hypothetical BG circuit. SNr neurons receive projections from the striatum and external globus pallidus, via the direct (D, striatonigral) and indirect (I, striatopallidal) pathways. Although these projections are GABAergic, the net effect on the $\mathrm{SNr}$ can be either inhibitory $(-$ ) or excitatory (disinhibitory, + ). Both types of signals, corresponding to a velocity error signal from the striatum, enter the SNr integrator, which is assumed to be leaky. Of course, direct and indirect pathways are not the only possible neural implementation of the proposed circuit. The key component is a phase splitter that generates opponent position reference signals from the SNr to the relevant downstream position controllers. Similar to reciprocal inhibition in the spinal cord, the proposed circuit generates opponent outputs for movement in any direction. For example, for upward movement, the indirect pathway neurons in the "Up" module increase the firing rate of $\mathrm{SNr}^{\text {" }} \mathrm{Up}^{+}$" neurons, whereas the direct pathway from the same module decreases the firing rate of $\mathrm{SNr}$ "Down ${ }^{+}$" neurons. The coordinated opponent outputs from this module generate movement in the desired direction. The rate of $\mathrm{SNr}$ output reflects the time integral of the striatal output. The striatum, especially the sensorimotor region, is hypothesized to contain at least four different modules, each responsible for movement in a specific direction. Because z-axis motion has not been measured, only four directions are illustrated here. Two of the four vector components are associated with any movement (e.g., upward and rightward), as movements usually have both horizontal and vertical components. The total output (number of spikes) from a particular striatal module represents the magnitude of the isolated position vector component, and the firing rate represents velocity (Kim et al., 2014). Using the outputs from different vector component modules, this circuit can perform vector addition to generate the resultant vector.

and speed can be represented by the firing rate of BG output neurons. Instead, we showed that $\mathrm{SNr}$ output at any given time reflects a distinct position, not movement amplitude or speed. The rate of change in nigral firing rate reflects movement velocity, whereas the total amount of change reflects movement amplitude.

The SNr neurons are probably affected by both direct and indirect pathways, which are simultaneously activated during movement (Cui et al., 2013). With overlapping excitatory inputs, the direct and indirect pathways can function as a phase splitter to generate antiphase signals from a common input. By exerting opposite effects on the $\mathrm{SNr}$, these pathways can generate opponent outputs. Our working model for how the BG circuit generates movement is shown in Figure 14.

\section{BG output and transition control}

As recently proposed, the $B G$ represent the level of transition control, which sends reference signals to lower-level position controllers for body configuration and orientation (Yin, 2010, 2014b). Such closed loop systems control their inputs by using the discrepancy between input and intrinsic reference to drive outputs. The error signal is self-reducing via negative feedback. When arranged in a cascade control hierarchy, the outputs of higher levels do not reach effectors directly but alter the reference signals of lower levels, thereby telling the lower systems to reach a specific level of input by varying output. The actual outputs from the lower level will depend on the comparison between its own local input and the descending reference. In a functional control system, the actual inputs will approach reference signals.

The transition control system is hierarchically higher than the position control system and generates movements by altering the reference signals of the position control system. The similarity between nigral firing rate and position coordinates suggests the presence of position control, which allows the maintenance of specific postures and head orientation. A descending reference signal from the BG output does not tell the lower level position control system how much output to produce, but how much input to sense. The high tonic "baseline" activity of nigral output neurons represents stable descending reference signal for orientation and configuration control in the tectum, brainstem, and thalamus (Fig. 14). It is associated with a stable posture. By changing this reference signal to new values, a succession of body configurations can be generated. The rate of change reflects movement velocity (i.e., rate of change in body configurations).

The $\mathrm{SNr}$ neurons receive inputs from other BG nuclei, such as the striatum. The firing rate of striatal output neurons is correlated with movement velocity (Kim et al., 2014). For example, with direct striatonigral projections, the nigral output could be produced by integrating velocity signals from the striatum. In an integrator, the rate of change of the output is proportional to the magnitude of the input. Thus, the striatal output, representing a velocity error signal, is integrated to yield the position-related signals from the nigral neurons. Integrators are common in analog computing and used in the output function of closed loop control systems to achieve stability and accuracy in control. Our results suggest that the BG output nuclei implement the output functions of transition controllers that control the rate of change in high-level perceptual inputs.

Despite different baseline firing rates of $\mathrm{SNr}$ neurons from a particular class, they often show comparable correlation with position coordinates, as each neuron shows a proportional change in firing rate for a given change in position (Fig. 11). Different neurons from a given class may receive similar inputs, and the computation appears to be multiplicative or divisive (Silver, 2010). A comparable effect may be achieved by integration, but without knowing the neural implementation of the integrator it is unclear how these computations are performed. These neurons are known to have a voltage-gated persistent sodium current, which allows persistent and high firing rates; they also express Type 3 transient receptor potential channels that can depolarize the cell at hyperpolarized membrane potentials (Atherton and Bevan, 2005; Zhou and Lee, 2011). How such intrinsic properties or recurrent circuit properties contribute to the relevant computations in vivo remains to be elucidated.

The sensorimotor BG circuit is hypothesized to be critical for the control of proprioceptive transitions (e.g., movement velocity) (Yin, 2014b). The SNr output can alter reference signal for body configurations and orientation, via projections to the tectum and other brainstem regions, such as the pedunculopontine tegmental nucleus (Robinson, 1972; Goodale and Murison, 1975; Waitzman et al., 1991; Takakusaki et al., 2004). These structures in turn can activate the reticulospinal pathway eventually to 
change the activation of the relevant muscles. For simple position control downstream, continuously varying outputs will be needed to command joint angle and muscle length controllers, and finally the muscle tension controllers (Yin, 2014a).

Our results have implications for understanding common clinical symptoms of movement disorders. The standard rate model conflates movement amplitude and speed, claiming that the excessively high firing rate of SNr/GPi neurons is responsible for parkinsonian symptoms like bradykinesia (slowed movement) and akinesia (Albin et al., 1989; DeLong, 1990). According to our model, these symptoms cannot be explained by excessive BG output; rather, they are attributed to a reduction in the rate of change in the firing rate of BG output nuclei. The chief effect of dopaminergic modulation is to regulate the velocity-related signals entering the integrator circuit, which generates changes in the reference signals for body configuration position control systems. Dopamine depletion, found in Parkinson's disease, reduces the magnitude of the signal entering the integrator, thus reducing the rate of change in the output (Yin, 2014b).

\section{References}

Albin RL, Young AB, Penney JB (1989) The functional anatomy of basal ganglia disorders. Trends Neurosci 12:366-375. CrossRef Medline

Atherton JF, Bevan MD (2005) Ionic mechanisms underlying autonomous action potential generation in the somata and dendrites of GABAergic substantia nigra pars reticulata neurons in vitro. J Neurosci 25:82728281. CrossRef Medline

Barter JW, Castro S, Sukharnikova T, Rossi MA, Yin HH (2014) The role of the substantia nigra in posture control. Eur J Neurosci 39:1465-1473. CrossRef Medline

Basso MA, Wurtz RH (2002) Neuronal activity in substantia nigra pars reticulata during target selection. J Neurosci 22:1883-1894. Medline

Bernstein N (1967) The coordination and regulation of movements. Oxford: Pergamon.

Bolam JP, Smith Y, Ingham CA, von Krosigk M, Smith AD (1993) Convergence of synaptic terminals from the striatum and the globus pallidus onto single neurones in the substantia nigra and the entopeduncular nucleus. Prog Brain Res 99:73-88. CrossRef Medline

Chevalier G, Deniau JM (1990) Disinhibition as a basic process in the expression of striatal functions. Trends Neurosci 13:277-280. CrossRef Medline

Chevalier G, Vacher S, Deniau JM (1984) Inhibitory nigral influence on tectospinal neurons, a possible implication of basal ganglia in orienting behavior. Exp Brain Res 53:320-326. Medline

Crutcher MD, Alexander GE (1990) Movement-related neuronal activity selectively coding either direction or muscle pattern in three motor areas of the monkey. J Neurophysiol 64:151-163. Medline

Cui G, Jun SB, Jin X, Pham MD, Vogel SS, Lovinger DM, Costa RM (2013) Concurrent activation of striatal direct and indirect pathways during action initiation. Nature 494:238-242. CrossRef Medline

DeLong MR (1990) Primate models of movement disorders of basal ganglia origin. Trends Neurosci 13:281-285. CrossRef Medline

DeLong MR, Alexander GE, Georgopoulos AP, Crutcher MD, Mitchell SJ, Richardson RT (1984) Role of basal ganglia in limb movements. Hum Neurobiol 2:235-244. Medline

Fan D, Rich D, Holtzman T, Ruther P, Dalley JW, Lopez A, Rossi MA, Barter JW, Salas-Meza D, Herwik S, Holzhammer T, Morizio J, Yin HH (2011) A wireless multi-channel recording system for freely behaving mice and rats. PLoS One 6:e22033. CrossRef Medline

Fan D, Rossi MA, Yin HH (2012) Mechanisms of action selection and timing in substantia nigra neurons. J Neurosci 32:5534-5548. CrossRef Medline

Freeze BS, Kravitz AV, Hammack N, Berke JD, Kreitzer AC (2013) Control of basal ganglia output by direct and indirect pathway projection neurons. J Neurosci 33:18531-18539. CrossRef Medline

Gerfen CR, Wilson CJ (1996) The basal ganglia. In: Handbook of chemical neuroanatomy (Swanson LW, Bjorklund A, Hokfelt T, eds), pp 371-468. Amsterdam: Elsevier.

Goodale MA, Murison RC (1975) The effects of lesions of the superior col- liculus on locomotor orientation and the orienting reflex in the rat. Brain Res 88:243-261. CrossRef Medline

Graybiel AM (1998) The basal ganglia and chunking of action repertoires. Neurobiol Learn Mem 70:119-136. CrossRef Medline

Gulley JM, Kuwajima M, Mayhill E, Rebec GV (1999) Behavior-related changes in the activity of substantia nigra pars reticulata neurons in freely moving rats. Brain Res 845:68-76. CrossRef Medline

Hikosaka O (2007) GABAergic output of the basal ganglia. Prog Brain Res 160:209-226. CrossRef Medline

Hikosaka O, Wurtz RH (1983) Visual and oculomotor functions of monkey substantia nigra pars reticulate: IV. Relation of substantia nigra to superior colliculus. J Neurophysiol 49:1285-1301. Medline

Hikosaka O, Takikawa Y, Kawagoe R (2000) Role of the basal ganglia in the control of purposive saccadic eye movements. Physiol Rev 80:953-978. Medline

Jin X, Costa RM (2010) Start/stop signals emerge in nigrostriatal circuits during sequence learning. Nature 466:457-462. CrossRef Medline

Kawagoe R, Takikawa Y, Hikosaka O (1998) Expectation of reward modulates cognitive signals in the basal ganglia. Nat Neurosci 1:411-416. CrossRef Medline

Kim N, Barter JW, Sukharnikova T, Yin HH (2014) Striatal firing rate reflects head movement velocity. Eur J Neurosci 40:3481-3490. CrossRef Medline

Lein ES, Hawrylycz MJ, Ao N, Ayres M, Bensinger A, Bernard A, Boe AF, Boguski MS, Brockway KS, Byrnes EJ, Chen L, Chen L, Chen TM, Chin MC, Chong J, Crook BE, Czaplinska A, Dang CN, Datta S, Dee NR, et al. (2007) Genome-wide atlas of gene expression in the adult mouse brain. Nature 445:168-176. CrossRef Medline

Matsumoto M, Hikosaka O (2007) Lateral habenula as a source of negative reward signals in dopamine neurons. Nature 447:1111-1115. CrossRef Medline

MinkJW (1996) The basal ganglia: focused selection and inhibition of competing motor programs. Prog Neurobiol 50:381-425. CrossRef Medline

Mitchell SJ, Richardson RT, Baker FH, DeLong MR (1987) The primate globus pallidus: neuronal activity related to direction of movement. Exp Brain Res 68:491-505. CrossRef Medline

Nicolelis MA (2007) Methods for neural ensemble recordings. Boca Raton, FL: CRC.

Parent A, Hazrati LN (1995) Functional anatomy of the basal ganglia: I. The cortico-basal ganglia-thalamo-cortical loop. Brain Res Brain Res Rev 20: 91-127. CrossRef Medline

Robinson DA (1972) Eye movements evoked by collicular stimulation in the alert monkey. Vision Res 12:1795-1808. CrossRef Medline

Romo R, Schultz W (1992) Role of primate basal ganglia and frontal cortex in the internal generation of movements: III. Neuronal activity in the supplementary motor area. Exp Brain Res 91:396-407. CrossRef Medline

Rossi MA, Fan D, Barter JW, Yin HH (2013a) Bidirectional modulation of substantia nigra activity by motivational state. PLoS One 8:e71598. CrossRef Medline

Rossi MA, Sukharnikova T, Hayrapetyan VY, Yang L, Yin HH (2013b) Operant self-stimulation of dopamine neurons in the substantia nigra. PLoS One 8:e65799. CrossRef Medline

Silver RA (2010) Neuronal arithmetic. Nat Rev Neurosci 11:474-489. CrossRef Medline

Takakusaki K, Saitoh K, Harada H, Kashiwayanagi M (2004) Role of basal ganglia-brainstem pathways in the control of motor behaviors. Neurosci Res 50:137-151. CrossRef Medline

Thompson RF, Thompson JK, Kim JJ, Krupa DJ, Shinkman PG (1998) The nature of reinforcement in cerebellar learning. Neurobiol Learn Mem 70:150-176. CrossRef Medline

Waitzman DM, Ma TP, Optican LM, Wurtz RH (1991) Superior colliculus neurons mediate the dynamic characteristics of saccades. J Neurophysiol 66:1716-1737. Medline

Yin HH (2010) The sensorimotor striatum is necessary for serial order learning. J Neurosci 30:14719-14723. CrossRef Medline

Yin HH (2014a) How the basal ganglia output generates behavior. Adv Neurosci 2014:768313. CrossRef

Yin HH (2014b) Action, time and the basal ganglia. Philos Trans R Soc B Biol Sci 369:20120473. CrossRef Medline

Yin HH, Knowlton BJ (2006) The role of the basal ganglia in habit formation. Nat Rev Neurosci 7:464-476. CrossRef Medline

Zhou FM, Lee CR (2011) Intrinsic and integrative properties of substantia nigra pars reticulata neurons. Neuroscience 198:69-94. CrossRef Medline 